\title{
Linear Fractional Transformations of Nevanlinna Functions Associated with a Nonnegative Operator
}

\author{
Jussi Behrndt • Seppo Hassi • Henk de Snoo • \\ Rudi Wietsma • Henrik Winkler
}

Received: 23 March 2011 / Accepted: 13 September 2011 / Published online: 13 October 2011

(C) The Author(s) 2011. This article is published with open access at Springerlink.com

\begin{abstract}
In the present paper a subclass of scalar Nevanlinna functions is studied, which coincides with the class of Weyl functions associated to a nonnegative symmetric operator of defect one in a Hilbert space. This class consists of all Nevanlinna functions
\end{abstract}

Dedicated to our friend Franek Szafraniec on the occasion of his seventieth birthday.

Communicated by Guest Editors L. Littlejohn and J. Stochel.

This research was supported by the grants from the Academy of Finland (project 139102) and the German Academic Exchange Service (DAAD project D/08/08852). The third author thanks the Deutsche Forschungsgemeinschaft (DFG) for the Mercator visiting professorship at the Technische Universität Berlin. The authors would like to thank also an anonymous referee on some constructive comments, especially for paying their attention to group actions.

J. Behrndt

Institut für Numerische Mathematik, Technische Universität Graz, Steyrergasse 30, 8010 Graz, Austria e-mail: behrndt@tugraz.at

S. Hassi · R. Wietsma

Department of Mathematics and Statistics, University of Vaasa, P.O. Box 700, 65101 Vaasa, Finland e-mail: sha@uwasa.fi

R. Wietsma

e-mail: rwietsma@uwasa.fi

H. de Snoo $(\varangle)$

Johann Bernoulli Institute for Mathematics and Computer Science, University of Groningen, P.O. Box 407, 9700 AK Groningen, The Netherlands e-mail: desnoo@math.rug.nl

H. Winkler

Institut für Mathematik, Technische Universität Ilmenau, Curiebau,

Weimarer Str. 25, 98693 Ilmenau, Germany

e-mail: henrik.winkler@tu-ilmenau.de 
that are holomorphic on $(-\infty, 0)$ and all those Nevanlinna functions that have one negative pole $a$ and are injective on $(-\infty, a) \cup(a, 0)$. These functions are characterized via integral representations and special attention is paid to linear fractional transformations which arise in extension and spectral problems of symmetric and selfadjoint operators.

\section{Introduction}

The class of Nevanlinna functions is intimately connected with selfadjoint operators and relations in Hilbert spaces, and therefore plays a key role in spectral analysis. For instance, the Titchmarsh-Weyl coefficients of real trace-normed $2 \times 2$ canonical systems on a halfline coincide with the Nevanlinna functions. Recall that a scalar function $Q$ is said to be a Nevanlinna function if it admits an integral representation of the form

$$
Q(\lambda)=\alpha+\beta \lambda+\int_{\mathbb{R}}\left(\frac{1}{s-\lambda}-\frac{s}{s^{2}+1}\right) d \sigma(s), \quad \lambda \in \mathbb{C} \backslash \mathbb{R},
$$

where $\alpha \in \mathbb{R}, \beta \geq 0$, and $\sigma$ is a nondecreasing function on $\mathbb{R}$ such that $\int_{\mathbb{R}} \frac{d \sigma(s)}{s^{2}+1}<\infty$. Various subclasses of Nevanlinna functions have been studied in the past, e.g., Kac, Stieltjes, and inverse Stieltjes functions in connection with spectral problems for strings; cf. [7, 17-21,23,24] and [3,8-10,15].

In the present paper the subclass of Nevanlinna functions is studied which consists of all Nevanlinna functions $Q$ which are holomorphic on $(-\infty, 0)$ and, in addition, of all those Nevanlinna functions $Q$ which have one pole at some point $a$ on the negative halfline and map $(-\infty, a) \cup(a, 0)$ injectively into $\mathbb{R}$. In terms of the (possibly improper) limits $Q(-\infty) \in[-\infty, \infty)$ and $Q(0) \in(-\infty, \infty]$ these Nevanlinna functions are divided into five separate subclasses I-V which are equivalently characterized via integral representations. In particular, all Stieltjes and inverse Stieltjes functions, as well as translations of those functions belong to the Nevanlinna subclasses I-V under consideration.

Suppose now that $Q$ is a Nevanlinna function of type $\mathrm{I}-\mathrm{V}$ and consider linear fractional transformations of the type

$$
Q_{\tau}(\lambda)=\frac{Q(\lambda)-\tau}{1+\tau Q(\lambda)}, \quad \tau \in \mathbb{R} \cup\{\infty\}, \quad \lambda \in \mathbb{C} \backslash \mathbb{R} .
$$

It turns out that also the transformed function $Q_{\tau}$ is a Nevanlinna function of type I-V and in terms of the parameter $\tau$ and the limits $Q(-\infty)$ and $Q(0)$ the precise type of $Q_{\tau}$ is determined. In particular, the function $Q$ gives rise to an interval of $\mathbb{R} \cup\{\infty\}$ so that for $\tau$ belonging to this interval or to its exterior the function $Q_{\tau}$ in (1.1) has precisely one pole on the negative halfaxis. When $\tau$ tends to the endpoints of this interval, the function $Q_{\tau}$ approximates in a certain sense the functions corresponding to the endpoints of this interval. At these "exceptional" values of $\tau$ the spectral measures of the functions $Q_{\tau}$ have a behaviour different from all the other transformations in (1.1). 
The above results are closely connected with extension theory. Let $S$ be a closed symmetric operator in a Hilbert space $\mathfrak{H}$ with defect numbers $(1,1)$. The selfadjoint extensions of $S$ can be described by a boundary triplet associated with $S^{*}$. All the boundary triplets associated with $S^{*}$ can be parametrized via operators which are unitary in a Kreŭn space sense. This parametrization gives rise to orbits of the Weyl function of the original boundary triplet; cf. [5]. The linear fractional transformation formula in (1.1) is just another form of this parametrization and the functions in (1.1) act as the Weyl functions of the relevant selfadjoint extensions. The class of Nevanlinna functions is invariant under these transformations. If the closed symmetric operator $S$ is nonnegative, then the corresponding Weyl functions have special characterizations, which are provided in this paper. It is shown that the Weyl functions corresponding to $S$ are Nevanlinna functions of type I-V, and that the set of all Nevanlinna functions of type I-V is invariant under the above transformations, although the subsets themselves are not. Moreover, each Nevanlinna function of type I-V can be obtained as a Weyl function of a nonnegative operator. The "exceptional" values of the parameter $\tau$ in (1.1) correspond to the Friedrichs and Krein-von Neumann extensions of $S$. Furthermore, stability results on the nonnegativity as well as a description of the possible negative eigenvalue of the selfadjoint extensions are obtained via (1.1).

Here is an enumeration of the contents of the paper. Section 2 contains an introduction to subclasses of Nevanlinna functions and their integral representations. Linear fractional transformations of these subclasses of Nevanlinna functions are studied in Sect. 3. In Sect. 4 the functions which are exceptional with respect to the linear fractional transformation are seen as limiting values of transformations with a pole on the negative halfaxis. Finally the connection with the extension theory of nonnegative symmetric operators in a Hilbert space with defect numbers $(1,1)$ is explained in Sect. 5.

\section{Some Classes of Nevanlinna Functions and Their Integral Representations}

In this section Nevanlinna functions are introduced and various subclasses are considered. These subclasses are defined by the requirement that the Nevanlinna function is holomorphic on the negative halfaxis with the possible exception of one pole at some point $a,-\infty<a<0$, and that it maps $(-\infty, a) \cup(a, 0)$ injectively into $\mathbb{R}$.

\subsection{Nevanlinna Functions}

The class $\mathbf{N}$ of Nevanlinna functions is the set of all scalar functions $Q$ which are holomorphic on $\mathbb{C} \backslash \mathbb{R}$ and which satisfy the symmetry and nonnegativity conditions:

$$
Q(\bar{\lambda})=\overline{Q(\lambda)} \text { and } \frac{\operatorname{Im} Q(\lambda)}{\operatorname{Im} \lambda} \geq 0, \quad \lambda \in \mathbb{C} \backslash \mathbb{R},
$$

cf. [6], [19]. Equivalently, the function $Q$ belongs to the class $\mathbf{N}$ if and only if there exist $\alpha \in \mathbb{R}, \beta \geq 0$, and a nondecreasing function $\sigma$ on $\mathbb{R}$ such that for $\lambda \in \mathbb{C} \backslash \mathbb{R}$

$$
Q(\lambda)=\alpha+\beta \lambda+\int_{\mathbb{R}}\left(\frac{1}{s-\lambda}-\frac{s}{s^{2}+1}\right) d \sigma(s), \int_{\mathbb{R}} \frac{d \sigma(s)}{1+s^{2}}<\infty .
$$


A direct calculation shows that $\alpha=\operatorname{Re} Q(i)$ and that

$$
\beta=\lim _{\lambda \rightarrow \infty} \frac{Q(\lambda)}{\lambda} \text { and } \lim _{\lambda \rightarrow c}(c-\lambda) Q(\lambda)=\sigma(c+)-\sigma(c-), \quad c \in \mathbb{R},
$$

where $\widehat{\rightarrow}$ stands for a non-tangential (sectorial) limit. The spectral function $\sigma$ in the integral representation of a function $Q \in \mathbf{N}$ is normalized by the condition

$$
\sigma(s)=\frac{\sigma(s+0)+\sigma(s-0)}{2}
$$

and fixed by $\sigma(0)=0$, so that $\sigma$ is uniquely determined. Under these circumstances the Stieltjes inversion formula holds:

$$
\sigma\left(s_{2}\right)-\sigma\left(s_{1}\right)=\lim _{\varepsilon \downarrow 0} \frac{1}{\pi} \int_{s_{1}}^{s_{2}} \operatorname{Im} Q(\lambda+i \varepsilon) d \lambda, \quad s_{1} \leq s_{2} .
$$

If $Q$ is holomorphic on an interval $(c, d) \subset \mathbb{R}$, i.e., if $\sigma$ is constant on $(c, d)$, then the derivative $Q^{\prime}$ has the integral representation

$$
Q^{\prime}(\lambda)=\beta+\int_{\mathbb{R} \backslash(c, d)} \frac{d \sigma(s)}{(s-\lambda)^{2}}, \quad c<\lambda<d,
$$

cf. (2.1). Hence, $Q$ is monotonically nondecreasing on any interval of the real line where $Q$ is holomorphic. In particular, if $Q$ is holomorphic on $(-\infty, c)$ for some $c \in \mathbb{R}$, then $Q$ has possibly improper limits for $\lambda \downarrow-\infty$ and for $\lambda \uparrow c$.

\subsection{Kac, Stieltjes, and Inverse Stieltjes Functions}

A Nevanlinna function $Q$ with the integral representation (2.1) is said to belong to the Kac class $($ at $\infty)$ if

$$
\beta=0 \text { and } \int_{\mathbb{R}} \frac{d \sigma(s)}{1+|s|}<\infty
$$

or equivalently, if the integral representation (2.1) of $Q$ takes the form

$$
Q(\lambda)=b+\int_{\mathbb{R}} \frac{d \sigma(s)}{s-\lambda}, \quad \int_{\mathbb{R}} \frac{d \sigma(s)}{1+|s|}<\infty,
$$

where by dominated convergence

$$
b=\lim _{\lambda \rightarrow \infty} Q(\lambda)=\alpha-\int_{\mathbb{R}} \frac{s}{1+s^{2}} d \sigma(s),
$$


see [19]. Likewise, a Nevanlinna function $Q$ is said to belong to the Kac class (at 0) if the Nevanlinna function $\lambda \mapsto-Q(1 / \lambda)$ belongs to the Kac class at $\infty$. It can be shown that a Nevanlinna function $Q$ with the integral representation (2.1) belongs to the Kac class (at 0) if and only if,

$$
\int_{-1}^{1} \frac{d \sigma(s)}{|s|}<\infty
$$

cf. [9, Proposition 3.1]. In particular, this condition implies that

$$
d \sigma(\{0\})=0 .
$$

Moreover, the integral representation (2.1) takes the form

$$
Q(\lambda)=\beta \lambda+L+\int_{\mathbb{R}}\left(\frac{1}{s-\lambda}-\frac{1}{s}\right) d \sigma(s),
$$

with

$$
L=\lim _{y \downarrow 0} Q(i y)=\alpha+\int_{\mathbb{R}} \frac{d \sigma(s)}{s\left(s^{2}+1\right)} .
$$

The relevant integrability conditions for (2.8) can be rewritten as

$$
\int_{\mathbb{R}} \frac{d \sigma(s)}{|s|(1+|s|)}<\infty
$$

In the case that $Q$ is holomorphic on an interval $(-\infty, c)$ for some $c \in \mathbb{R}$, the Kac class $($ at $\infty$ ) can also be characterized by a single limit (see e.g. [16, Corollary 3.4]); here a direct proof is included for completeness. In the following $Q(\infty)$ denotes the sectorial limit of $Q(\lambda)$ for $\lambda \widehat{\rightarrow} \infty$; it is equal to the limit value $Q(-\infty)=\lim _{\lambda \downarrow-\infty} Q(\lambda)$ on the real line, if $Q$ is holomorphic on an interval $(-\infty, c)$.

Proposition 2.1 Let $Q \in \mathbf{N}$ and assume that $Q$ is holomorphic on $(-\infty, c)$. Then

$$
\lim _{\lambda \downarrow-\infty} Q(\lambda) \in \mathbb{R}
$$

if and only if $Q$ belongs to the Kac class (at $\infty)$ or, equivalently, the integral representation (2.1) of $Q$ takes the form

$$
Q(\lambda)=b+\int_{[c, \infty)} \frac{d \sigma(s)}{s-\lambda}, \int_{[c, \infty)} \frac{d \sigma(s)}{1+|s|}<\infty,
$$


where

$$
b=\lim _{\lambda \downarrow-\infty} Q(\lambda)=\alpha-\int_{[c, \infty)} \frac{s}{s^{2}+1} d \sigma(s) .
$$

Proof Since $Q$ is holomorphic on $(-\infty, c)$, it is monotonically nondecreasing on $(-\infty, c)$; see (2.3). Hence, $Q(-\infty)=\lim _{\lambda \downarrow-\infty} Q(\lambda)$ exists in $\mathbb{R} \cup\{-\infty\}$. If $\lim _{\lambda \downarrow-\infty} Q(\lambda) \in \mathbb{R}$, then necessarily $\beta=0$; see (2.2). On the other hand, by letting $\lambda \downarrow-\infty$ in (2.1) (with $\lambda<c$ ) it follows by monotone convergence that the equality in (2.9) holds and clearly $b \in \mathbb{R}$ if and only if $\int_{[c, \infty)} \frac{s}{s^{2}+1} d \sigma(s)<\infty$. Therefore (2.4) follows, i.e. $Q$ belongs to the Kac class (at $\infty$ ).

Conversely, if $Q$ belongs to the Kac class (at $\infty$ ) then it follows from (2.5) (by dominated convergence) that $\lim _{\lambda \downarrow-\infty} Q(\lambda)=\lim _{\lambda} \widehat{\rightarrow \infty} Q(\lambda) \in \mathbb{R}$.

Similarly, if $Q$ is holomorphic on an interval $(c, 0)$ with $c<0$, then the Kac class (at 0 ) can be characterized by applying similar arguments as in the proof of Proposition 2.1. In the following $Q(0)$ denotes the sectorial limit $Q(0)=\lim _{y \downarrow 0} Q(i y)$, which is equal to $Q(0)=\lim _{\lambda \uparrow 0} Q(\lambda)$ if $Q$ is holomorphic on an interval $(c, 0)$ with $c<0$.

Proposition 2.2 Let $Q \in \mathbf{N}$ and suppose that $Q$ is holomorphic on $(c, 0)$ for some $c<0$. Then

$$
\lim _{\lambda \uparrow 0} Q(\lambda) \in \mathbb{R}
$$

if and only if $Q$ belongs to the Kac class (at 0) or, equivalently, the integral representation (2.1) of $Q$ takes the form

$$
Q(\lambda)=\beta \lambda+L+\int_{\mathbb{R} \backslash(c, 0]}\left(\frac{1}{s-\lambda}-\frac{1}{s}\right) d \sigma(s), \int_{\mathbb{R} \backslash(c, 0]} \frac{d \sigma(s)}{|s|(1+|s|)}<\infty,
$$

where

$$
L=\lim _{\lambda \uparrow 0} Q(\lambda)=\alpha+\int_{\mathbb{R} \backslash(c, 0]} \frac{d \sigma(s)}{s\left(s^{2}+1\right)} .
$$

A combination of Propositions 2.1 and 2.2 gives also the following observation: if $Q \in \mathbf{N}$ is holomorphic on $(-\infty, c) \cup(d, 0)$ for some $c, d<0$, then

$$
\lim _{\lambda \downarrow-\infty} Q(\lambda) \in \mathbb{R} \text { and } \lim _{\lambda \uparrow 0} Q(\lambda) \in \mathbb{R} \Leftrightarrow \int_{\mathbb{R}} \frac{d \sigma(s)}{|s|}<\infty,
$$

equivalently, $Q$ belongs to the Kac class, both at $\infty$ and 0 . 
Particular examples of Kac functions which satisfy the conditions of Propositions 2.1 and 2.2 are the so-called Stieltjes and inverse Stieltjes functions, respectively. Recall that a Nevanlinna function $Q$ is said to belong to the class of Stieltjes or inverse Stieltjes functions, denoted by $\mathbf{S}$ and $\mathbf{S}^{-1}$, if and only if $Q$ is holomorphic on $(-\infty, 0)$ and $Q$ is nonnegative or nonpositive on $(-\infty, 0)$, respectively. As a consequence of Propositions 2.1 and 2.2 one obtains the well-known integral representations of Stieltjes and inverse Stieltjes functions in [19].

Corollary 2.3 Let $Q \in \mathbf{N}$ be a Nevanlinna function. Then the following holds:

(i) $Q$ belongs to the Stieltjes class $\mathbf{S}$ if and only if it has an integral representation of the form

$$
Q(\lambda)=b+\int_{[0, \infty)} \frac{d \sigma(s)}{s-\lambda}, \quad \int_{[0, \infty)} \frac{d \sigma(s)}{1+s}<\infty
$$

where $0 \leq b=\lim _{\lambda \downarrow-\infty} Q(\lambda)$;

(ii) $Q$ belongs to the inverse Stieltjes class $\mathbf{S}^{-1}$ if and only if it has an integral representation of the form

$$
Q(\lambda)=\beta \lambda+L+\int_{(0, \infty)}\left(\frac{1}{s-\lambda}-\frac{1}{s}\right) d \sigma(s), \int_{(0, \infty)} \frac{d \sigma(s)}{s+s^{2}}<\infty
$$

where $0 \geq L=\lim _{\lambda \uparrow 0} Q(\lambda)$.

\subsection{Nevanlinna Functions of Type I-V}

The subclass of Nevanlinna functions introduced in the next definition plays the main role in the present paper. In particular, the Stieltjes and inverse Stieltjes functions are contained in the class in Definition 2.4 below. Recall first that a Nevanlinna function $Q$ which is holomorphic on $(-\infty, 0)$ with the possible exception of at most one point $a \in(-\infty, 0)$ admits possibly improper limits at $-\infty$ and 0 ,

$$
Q(-\infty):=\lim _{\lambda \downarrow-\infty} Q(\lambda) \in \mathbb{R} \cup\{-\infty\} \quad \text { and } \quad Q(0):=\lim _{\lambda \uparrow 0} Q(\lambda) \in \mathbb{R} \cup\{+\infty\}
$$

and that $a$ is necessarily a pole if it is not a point of holomorphy of $Q$. If $a$ is a pole, then $Q$ has one-sided improper limits at $a$ :

$$
\lim _{\lambda \downarrow a} Q(\lambda)=-\infty \quad \text { and } \quad \lim _{\lambda \uparrow a} Q(\lambda)=+\infty
$$

Definition 2.4 Let $Q$ be a nonconstant Nevanlinna function. Then $Q$ is said to be of type

I if $Q$ is holomorphic on $\mathbb{C} \backslash[0, \infty)$ with finite limits $Q(-\infty) \in \mathbb{R}$ and $Q(0) \in \mathbb{R}$; 
II if $Q$ is holomorphic on $\mathbb{C} \backslash[0, \infty)$ with a finite limit $Q(-\infty) \in \mathbb{R}$ and the improper limit $Q(0)=+\infty$;

III if $Q$ is holomorphic on $\mathbb{C} \backslash[0, \infty)$ with the improper limit $Q(-\infty)=-\infty$ and a finite limit $Q(0) \in \mathbb{R}$;

IV if $Q$ is holomorphic on $\mathbb{C} \backslash[0, \infty)$ with the improper limits $Q(-\infty)=-\infty$ and $Q(0)=+\infty$;

$\mathrm{V}$ if $Q$ is holomorphic on $\mathbb{C} \backslash[0, \infty)$ except for a pole $a$ in $(-\infty, 0)$ and $Q$ maps the set $(-\infty, a) \cup(a, 0)$ injectively into $\mathbb{R}$.

Observe that a Nevanlinna function $Q$ of type I-IV maps $(-\infty, 0)$ injectively into $\mathbb{R}$, so that any Nevanlinna function of type $\mathrm{I}-\mathrm{V}$ is injective on $(-\infty, 0)$ or the union $(-\infty, a) \cup(a, 0)$. Note also that for a Nevanlinna function $Q$ of type $\mathrm{V}$ the limits $Q(-\infty)$ and $Q(0)$ are finite, and that $Q(-\infty) \geq Q(0)$ holds. The class V will be split up in two disjoint subclasses: A nonconstant Nevanlinna function $Q$ is said to be of type

$\mathrm{V}^{\prime}$ if $Q$ is of type $\mathrm{V}$ and $Q(-\infty)>Q(0)$ holds;

$\mathrm{V}^{\prime \prime}$ if $Q$ is of type $\mathrm{V}$ and $Q(-\infty)=Q(0)$ holds.

A simple characterization of Nevanlinna functions of type $\mathrm{I}-\mathrm{V}$ is given in the next lemma.

Lemma 2.5 Let $Q$ be a Nevanlinna function which is holomorphic on $(-\infty, 0)$ with the possible exception of one pole on $(-\infty, 0)$. Then $Q$ is of type $\mathrm{I}-\mathrm{V}$ if and only if for each $c \in \mathbb{R}$ the shifted function $Q+c$ has at most one zero in $(-\infty, 0)$.

Next the Nevanlinna functions of type I-V are characterized by means of integral representations.

Proposition 2.6 Let $Q$ be a nonconstant Nevanlinna function which is holomorphic on $(-\infty, 0)$ with the possible exception of at most one point $a \in(-\infty, 0)$, and let $Q(0)$ and $Q(-\infty)$ be as in (2.10). Then

(i) $Q$ is of type I if and only if the integral representation of $Q$ is of the form

$$
Q(\lambda)=Q(-\infty)+\int_{(0, \infty)} \frac{d \sigma(s)}{s-\lambda},
$$

where $Q(-\infty) \in \mathbb{R}$ and $\int_{(0, \infty)} \frac{d \sigma(s)}{s}<\infty$;

(ii) $Q$ is of type II if and only if the integral representation of $Q$ is of the form

$$
Q(\lambda)=Q(-\infty)+\int_{[0, \infty)} \frac{d \sigma(s)}{s-\lambda},
$$

where $Q(-\infty) \in \mathbb{R}, \int_{[0, \infty)} \frac{d \sigma(s)}{1+s}<\infty$ and $\int_{[0, \infty)} \frac{d \sigma(s)}{s}=\infty ;$

(iii) $Q$ is of type III if, and only if the integral representation of $Q$ is of the form

$$
Q(\lambda)=\beta \lambda+Q(0)+\int_{(0, \infty)}\left(\frac{1}{s-\lambda}-\frac{1}{s}\right) d \sigma(s),
$$


where $Q(0) \in \mathbb{R}, \int_{(0, \infty)} \frac{d \sigma(s)}{s+s^{2}}<\infty$, and

$$
\beta>0 \text { or } \int_{(0, \infty)} \frac{d \sigma(s)}{1+s}=\infty
$$

(iv) $Q$ is of type IV if and only if the integral representation of $Q$ is of the form

$$
Q(\lambda)=\beta \lambda+\alpha+\int_{[0, \infty)}\left(\frac{1}{s-\lambda}-\frac{s}{s^{2}+1}\right) d \sigma(s),
$$

where $\int_{[0, \infty)} \frac{d \sigma(s)}{1+s^{2}}<\infty, \int_{[0, \infty)} \frac{d \sigma(s)}{s(1+s)}=\infty$, and

$$
\beta>0 \text { or } \int_{[0, \infty)} \frac{d \sigma(s)}{1+s}=\infty
$$

(v) $Q$ is of type $\mathrm{V}$ if and only if the integral representation of $Q$ is of the form

$$
Q(\lambda)=Q(-\infty)+\frac{m}{a-\lambda}+\int_{(0, \infty)} \frac{d \sigma(s)}{s-\lambda},
$$

where $m>0, \int_{[0, \infty)} \frac{d \sigma(s)}{s}<\infty$, and

$$
Q(-\infty)-Q(0)=-\left(\frac{m}{a}+\int_{(0, \infty)} \frac{d \sigma(s)}{s}\right) \geq 0
$$

or equivalently,

$$
m \geq-a \int_{(0, \infty)} \frac{d \sigma(s)}{s} .
$$

Proof (i) For a Nevanlinna function $Q$ of type I Proposition 2.1 implies that $Q$ admits an integral representation of the form

$$
Q(\lambda)=Q(-\infty)+\int_{[0, \infty)} \frac{d \sigma(s)}{s-\lambda}
$$

where $Q(-\infty) \in \mathbb{R}$ and $\int_{[0, \infty)} \frac{d \sigma(s)}{1+s}<\infty$. Furthermore, by Proposition 2.2

$$
\int_{(0, \infty)} \frac{d \sigma(s)}{s}<\infty
$$


and, in particular, there is no point mass of $\sigma$ at 0 . This and the integral representation (2.13) imply the integral representation of $Q$ in (i). Conversely, if $Q$ admits the integral representation in (i), then $\lim _{\lambda \uparrow 0} Q(\lambda) \in \mathbb{R}$ follows from the monotone convergence theorem. Together with $Q(-\infty) \in \mathbb{R}$ one concludes that $Q$ is a Nevanlinna function of type I.

(ii), (iii), and (iv) can be shown with similar arguments by applying Proposition 2.1 and Proposition 2.2. The details are left to the reader.

(v) Suppose that $Q$ is a Nevanlinna function of type V. Then the limit values $Q(-\infty)$ and $Q(0)$ are finite and the integral representation in Proposition 2.1 reduces to

$$
Q(\lambda)=Q(-\infty)+\frac{m}{a-\lambda}+\int_{[0, \infty)} \frac{d \sigma(s)}{s-\lambda}
$$

where $Q(-\infty) \in \mathbb{R}, m>0$ and $\int_{[0, \infty)} \frac{d \sigma(s)}{1+s}<\infty$. Moreover, Proposition 2.2 implies the integrability condition (2.14), so that there is no point mass of $\sigma$ at 0 and hence (2.15) coincides with the integral representation in (v). Since $Q$ maps the intervals $(-\infty, a)$ and $(a, 0)$ injectively into $\mathbb{R}$ the inequality $Q(-\infty) \geq Q(0)$ holds and the formula (2.11) for $Q(-\infty)-Q(0)$ is obtained directly from the given integral representation of $Q$. The condition (2.12) is equivalent to $Q(-\infty)-Q(0) \geq 0$, since $a<0$. Conversely, if $Q$ admits the integral representation in (v) with the additional properties, then it follows directly that $Q$ is a Nevanlinna function of type V.

Part (v) in Proposition 2.6 shows that a Nevanlinna function $Q$ which is holomorphic on $(-\infty, 0)$ with the exception of a pole at $a$ is of the type $\mathrm{V}$ if and only if the point mass $m$ that is concentrated at the point $a<0$ is sufficiently large, see (2.12); an operator-theoretic interpretation of this is given in Sect. 5.

Remark 2.7 Observe from Proposition 2.6 that only the functions in classes I, II, and $\mathrm{V}$ belong to the Kac class at $\infty$, whereas only the functions in classes I, III, and V belong to the Kac class at 0; cf. (2.4) and (2.7).

\section{Linear Fractional Transformations}

For a nonconstant Nevanlinna function $Q$, the linear fractional transformation $Q_{\tau}$ of $Q$ is defined as

$$
Q_{\tau}(\lambda)=\frac{Q(\lambda)-\tau}{1+\tau Q(\lambda)}=\frac{1}{\tau}-\frac{\tau^{2}+1}{\tau^{2}} \frac{1}{Q(\lambda)+1 / \tau}, \quad \tau \in \mathbb{R} \cup\{\infty\},
$$

with the interpretation that $Q_{\infty}(\lambda)=-1 / Q(\lambda)$. Clearly, each $Q_{\tau}$ is a Nevanlinna function. Notice that compositions of linear fractional transformations produce just a linear fractional transformation of the original function:

$$
\left(Q_{\tau}\right)_{\sigma}(\lambda)=Q_{\frac{\sigma+\tau}{1-\sigma \tau}}(\lambda)
$$


where the index of $Q$ should be properly understood; for further details, see Sect. 3.4 below. In the linear fractional transformation (3.1) there is continuity in the parameter as shown in the next lemma.

Lemma 3.1 Let $Q$ be a nonconstant Nevanlinna function and let the function $Q_{\tau}$ be defined by (3.1). Then $Q_{\tau}$ converges uniformly to $Q_{\rho}$ on compact sets in $\mathbb{C} \backslash \mathbb{R}$ as $\tau$ converges to $\rho$ when $\tau, \rho \in \mathbb{R} \cup\{\infty\}$.

Proof Since $Q$ is nonconstant, $Q(\lambda)$ is nonreal if $\lambda \in \mathbb{C} \backslash \mathbb{R}$, i.e. $1 /(Q(\lambda)+a)$ is bounded for all $a \in \mathbb{R}$ on compact sets in $\mathbb{C} \backslash \mathbb{R}$. Therefore by (3.1)

$$
Q_{\tau}(\lambda)-Q_{\rho}(\lambda)=\frac{\rho-\tau}{\tau \rho} \frac{Q(\lambda)^{2}+1}{(Q(\lambda)+1 / \tau)(Q(\lambda)+1 / \rho)},
$$

which shows that $Q_{\tau}(\lambda)$ converges uniformly to $Q_{\rho}(\lambda)$ on compact sets in $\mathbb{C} \backslash \mathbb{R}$, when $\tau, \rho \in \mathbb{R}$. The other cases are shown in a similar way.

The linear fractional transformation (3.1) of a Kac function belongs to the same class for all $\tau \in \mathbb{R} \cup\{\infty\}$ with one exceptional value; see [9,10,12]. More precisely, if $Q$ is a function in the Kac class at $\infty$, then all functions $Q_{\tau}$ in (3.1) belong to the Kac class at $\infty$ with the exception of the value $\tau=-1 / Q(\infty)$. Likewise, if $Q$ is a function in the Kac class at 0 , then all functions $Q_{\tau}$ in (3.1) belong to the Kac class at 0 with the exception of the value $\tau=-1 / Q(0)$.

In this section the linear fractional transformations $Q_{\tau}$ of a Nevanlinna function $Q$ of type I-V will be investigated. The limit values of the function $Q$ at $-\infty$ and 0 play a crucial role. Therefore, in what follows, the short-hand notations $b$ and $L$ denote these possibly improper limits:

$$
b:=\lim _{\lambda \downarrow-\infty} Q(\lambda) \in \mathbb{R} \cup\{-\infty\} \quad \text { and } \quad L:=\lim _{\lambda \uparrow 0} Q(\lambda) \in \mathbb{R} \cup\{+\infty\} .
$$

\subsection{Poles and Zeros of Linear Fractional Transformations}

Let $Q$ be a Nevanlinna function of type I-V and consider the linear fractional transformations $Q_{\tau}$ of $Q$ in (3.1). The values that $Q$ takes on the negative real line determine the zeros and poles of the transformations $Q_{\tau}$ on the interval $(-\infty, 0)$. Observe that for $\lambda \in(-\infty, 0)$

$$
Q_{\tau}(\lambda)=0 \Leftrightarrow Q(\lambda)=\tau \text { and }\left(Q_{\tau}(\lambda)\right)^{-1}=0 \quad \Leftrightarrow \quad Q(\lambda)=-1 / \tau .
$$

Lemma 3.2 Let $Q$ be a Nevanlinna function of the type I-V. Then the following holds for $\tau \in \mathbb{R} \cup\{\infty\}$ :

(i) $Q_{\tau}$ has a (necessarily unique) zero on $(-\infty, 0)$ precisely when

$$
\begin{cases}b<\tau<L, & \text { if } Q \text { is of type } \mathrm{I}-\mathrm{IV} \\ -\infty<\tau<L \quad \text { or } \quad b<\tau \leq \infty, & \text { if } Q \text { is of type } \mathrm{V}\end{cases}
$$


(ii) $Q_{\tau}$ has a (necessarily unique) pole on $(-\infty, 0)$ precisely when

$$
\begin{cases}b<-1 / \tau<L, & \text { if } Q \text { is of type } \mathrm{I}-\mathrm{IV} \\ -\infty<-1 / \tau<L \quad \text { or } \quad b<-1 / \tau \leq \infty, & \text { if } Q \text { is of type } \mathrm{V}\end{cases}
$$

Proof Since the proof for the statements (i) and (ii) are similar, only (i) will be shown here.

If $Q$ is of type I-IV, then $Q$ is monotonously increasing on $(-\infty, 0)$ and hence $Q$ maps $(-\infty, 0)$ bijectively onto the interval $(b, L)$. There $Q_{\tau}$ has a (necessarily unique) zero for $b<\tau<L$, see (3.4). If $Q$ is of type $\mathrm{V}$ and $a \in(-\infty, 0)$ is the pole of $Q$, then $Q$ is monotonously increasing on $(-\infty, a)$ and $(a, 0)$, and the injectivity condition in Definition $2.4 \mathrm{~V}$ implies that $Q$ maps $(-\infty, a) \cup(a, 0)$ bijectively onto the set $(-\infty, L) \cup(b,+\infty)$. Hence $Q_{\tau}$ has a (necessarily unique) zero for $-\infty<\tau<L$ or $b<\tau<\infty$ in this case. Furthermore, $Q_{\infty}=-1 / Q$ also has a zero, namely at $a$.

Corollary 3.3 Let $Q$ be a Nevanlinna function of the type $\mathrm{V}$. Then the transformation $Q_{\tau}$ is holomorphic on $(-\infty, 0)$ if and only if $L \leq-1 / \tau \leq b$. If, in particular, $Q$ is of type $\mathrm{V}^{\prime \prime}$, i.e. $L=b$, then $Q_{\tau}$ is holomorphic on $(-\infty, 0)$ only for the value $\tau=-1 / L=-1 / b$.

Proposition 3.4 Let $Q$ be a Nevanlinna function of the type I-V. Then

(i) if $Q$ is of type $\mathrm{I}-\mathrm{IV}$, then $Q_{\tau}$ has precisely one zero and one pole on $(-\infty, 0)$ if and only if

$$
b<\tau<-\frac{1}{L} \leq 0 \text { or } 0 \leq-\frac{1}{b}<\tau<L
$$

(ii) if $Q$ is of type $\mathrm{V}$, then $Q_{\tau}$ is holomorphic on $(-\infty, 0)$ and has no zeros on $(-\infty, 0)$ if and only if

$$
-\infty<L \leq \tau \leq-\frac{1}{b}<0 \text { or } 0<-\frac{1}{L} \leq \tau \leq b<\infty
$$

Proof (i) If $Q$ is of type I-IV, then $Q$ is holomorphic on $(-\infty, 0)$ and hence $b<L$. By Lemma $3.2 Q_{\tau}$ has one zero and one pole on $(-\infty, 0)$ if and only if $b<\tau<L$ and $b<-1 / \tau<L$. In this case, necessarily $\tau \in \mathbb{R} \backslash\{0\}$ and, moreover, $-\infty \leq b<0$ and $0<L \leq \infty$. Now, if $\tau<0$ then the four inequalities are equivalent to $b<\tau<$ $-\frac{1}{L} \leq 0$, and if $\tau>0$ then the four inequalities are equivalent to $-\frac{1}{b}<\tau<L$.

(ii) If $Q$ is of type $\mathrm{V}$, then $b, L \in \mathbb{R}$ and $L \leq b$. By Lemma $3.2 Q_{\tau}$ has no zeros and no poles on $(-\infty, 0)$ if and only if $L \leq \tau \leq b$ and $L \leq-1 / \tau \leq b$. Hence, necessarily $\tau \in \mathbb{R} \backslash\{0\}$ and, moreover, $-\infty<L<0$ and $0<b<\infty$. Now, if $\tau<0$ then the four inequalities are equivalent to $L \leq \tau \leq-\frac{1}{b}<0$, and if $\tau>0$ then the four inequalities are equivalent to $0<-\frac{1}{L} \leq \tau \leq b$. 


\subsection{Linear Fractional Transformations of Nevanlinna Functions of Type I-V}

For the transformation $Q_{\tau}, \tau \in \mathbb{R} \cup\{\infty\}$, of $Q$ introduce the notation

$$
b_{\tau}=\lim _{\lambda \downarrow-\infty} Q_{\tau}(\lambda) \text { and } L_{\tau}=\lim _{\lambda \uparrow 0} Q_{\tau}(\lambda),
$$

so that $b=b_{0}$ and $L=L_{0}$. These limits exist as improper limits, $b_{\tau} \in \mathbb{R} \cup\{-\infty\}$ and $L_{\tau} \in \mathbb{R} \cup\{+\infty\}$, since the transformations $Q_{\tau}$ are Nevanlinna functions with at most one isolated pole on $(-\infty, 0)$; cf. Lemma 3.2.

It is clear from (3.1) that the following connections exist between $b$ and $b_{\tau}$, and between $L$ and $L_{\tau}$ for $\tau \in(\mathbb{R} \backslash\{0\}) \cup\{\infty\}$ :

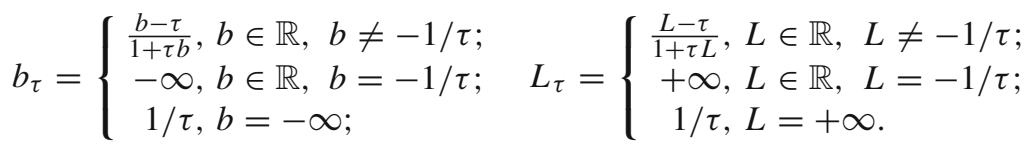

Observe, that $b_{\tau}=0$ if and only if $\tau=b$ and that $b_{\tau}=-\infty$ if and only if $\tau=-1 / b$; for all other values of $\tau \in \mathbb{R} \cup\{\infty\}$ one has $b_{\tau} \in \mathbb{R} \backslash\{0\}$. Clearly, the same holds for $L_{\tau}$.

These properties can be specified further for Nevanlinna functions of the type I-V. First of all, as the following lemma shows the class of Nevanlinna functions of type $\mathrm{I}-\mathrm{V}$ is stable under the linear fractional transformations (3.1).

Lemma 3.5 Let $Q$ be a Nevanlinna function of type $\mathrm{I}-\mathrm{V}$. Then for all $\tau \in \mathbb{R} \cup\{\infty\}$ the linear fractional transformation $Q_{\tau}$ in (3.1) is a Nevanlinna function of type I-V, too.

Proof Since each linear fractional transformation $Q_{\tau}, \tau \in \mathbb{R} \cup\{\infty\}$, is a nonconstant Nevanlinna function it suffices to verify that $Q_{\tau}$ is holomorphic on $(-\infty, 0)$ with the possible exception of at most one pole $a_{\tau} \in(-\infty, 0)$ in which case the injectivity condition in V holds. Observe first that by Lemma $3.2 Q_{\tau}$ is holomorphic on $(-\infty, 0)$ (and hence of the type I-IV) or $Q_{\tau}$ has a unique pole $a_{\tau} \in(-\infty, 0)$. In the latter case $Q_{\tau}$ maps $\left(-\infty, a_{\tau}\right) \cup\left(a_{\tau}, 0\right)$ injectively into $\mathbb{R}$ as otherwise (3.1) implies that $Q$ is not injective on $(-\infty, 0)$ (if $Q$ is of type I-IV) or $(-\infty, a) \cup(a, 0)$ (if $Q$ is of type $\mathrm{V}$ with pole $a \in(-\infty, 0))$.

More precise information on the linear fractional transformations (3.1) of Nevanlinna functions of the type $\mathrm{I}-\mathrm{V}$ is summarized in the next proposition and corollary; operator-theoretic interpretations appear in Corollaries 5.4 and 5.6.

Proposition 3.6 Let $Q$ be a Nevanlinna function of type $\mathrm{I}-\mathrm{V}$, let $Q_{\tau}$ be given by (3.1), and let $b_{\tau}$ and $L_{\tau}$ be as in (3.5) with $\tau \in \mathbb{R} \cup\{\infty\}$. Then the following cases can be distinguished:

(i) Let $Q$ be of type I-III. Then

- $Q_{\tau}$ is of type I if $-1 / \tau<b$ or $-1 / \tau>L$;

$-Q_{\tau}$ is of type II if $\tau=-1 / L$;

$-Q_{\tau}$ is of type III if $\tau=-1 / b$; 
- $Q_{\tau}$ is of type $\mathrm{V}^{\prime}$ if $b<-1 / \tau<L$.

(ii) Let $Q$ be of type IV. Then

- $Q_{\tau}$ is of the type IV for $\tau=0$;

- $Q_{\tau}$ is of the type $\mathrm{V}^{\prime \prime}$ for all $\tau \neq 0, \tau \in \mathbb{R} \cup\{\infty\}$.

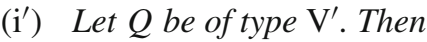

- $Q_{\tau}$ is of type I if $L<-1 / \tau<b$;

$-Q_{\tau}$ is of type II if $\tau=-1 / L$;

$-Q_{\tau}$ is of type III if $\tau=-1 / b$;

- $Q_{\tau}$ is of type $\mathrm{V}^{\prime}$ if $-1 / \tau<L$ or $-1 / \tau>b$.

(ii") Let $Q$ be of type $\mathrm{V}^{\prime \prime}$. Then

- $Q_{\tau}$ is of type IV if $\tau=-1 / L=-1 / b$;

- $Q_{\tau}$ is of type $\mathrm{V}^{\prime \prime}$ for all $\tau \neq-1 / L=-1 / b, \tau \in \mathbb{R} \cup\{\infty\}$.

The following corollary generalizes Lemma 3.5. It shows that with respect to the linear fractional transform (3.1) the Nevanlinna functions of type I-V split up into two mutually exclusive subclasses.

Corollary 3.7 If $Q$ is a Nevanlinna function of type I-III or $\mathrm{V}^{\prime}$, then the same holds for $Q_{\tau}, \tau \in \mathbb{R} \cup\{\infty\}$. If $Q$ is a Nevanlinna function of type IV or $\mathrm{V}^{\prime \prime}$, then the same holds for $Q_{\tau}, \tau \in \mathbb{R} \cup\{\infty\}$.

Proof of Proposition 3.6 First consider the case (ii). If $Q$ is of type IV, then by Lemma $3.2 Q_{\tau}$ has a (unique) pole on $(-\infty, 0)$ for all $\tau \neq 0, \tau \in \mathbb{R} \cup\{\infty\}$, and hence $Q_{\tau}$ is of type $\mathrm{V}$. Since $b=-\infty$ and $L=+\infty$, the formulas in (3.5) show that $b_{\tau}=1 / \tau \in \mathbb{R}$ and $L_{\tau}=1 / \tau \in \mathbb{R}$ for $\tau \neq 0$. Therefore, the equality $L_{\tau}=b_{\tau}$ holds for all $\tau \neq 0$, i.e. $Q_{\tau}$ is of type $\mathrm{V}^{\prime \prime}$.

Next consider the case (ii"). If $Q$ is of type $\mathrm{V}^{\prime \prime}$, then by Corollary $3.3 Q_{\tau}$ is holomorphic on $(-\infty, 0)$ if and only if $\tau=-1 / L=-1 / b$. Now the formulas in (3.5) show that for this value of $\tau$ one has $b_{\tau}=-\infty$ and $L_{\tau}=+\infty$. Hence, for $\tau=-1 / L=-1 / b$ the transformation $Q_{\tau}$ is of type IV. On the other hand, if $\tau \neq-1 / L(=-1 / b)$, then by Lemma $3.2 Q_{\tau}$ has a (unique) pole on $(-\infty, 0)$. Moreover, since $L=b$ it is clear from (3.5) that $L_{\tau}=b_{\tau}$ holds, that is, $Q_{\tau}$ is of type $\mathrm{V}^{\prime \prime}$.

The statements in (i) and (i') can be proved similarly by means of Lemma 3.2 and the formulas in (3.5).

\subsection{Exceptional Linear Fractional Transformations of Nevanlinna Functions of Type I-V}

Recall that the Nevanlinna functions of type I, II, and V belong to the Kac class at $\infty$ and that the Nevanlinna functions of type I, III, and V belong to the Kac class at 0 . The results in Proposition 3.6 will now be discussed from the point of view of Kac classes and the corresponding exceptional functions. The exceptional functions are related to the Friedrichs and Kreĭn-von Neumann extensions of a nonnegative operator with defect $(1,1)$; see Sect. 5 .

Let $Q$ be a Nevanlinna function of type I (so that $-\infty<b<L<\infty$ ). The behavior of the linear fractional transform $Q_{\tau}$ is sketched in Fig. 1. If $b<L$ have 


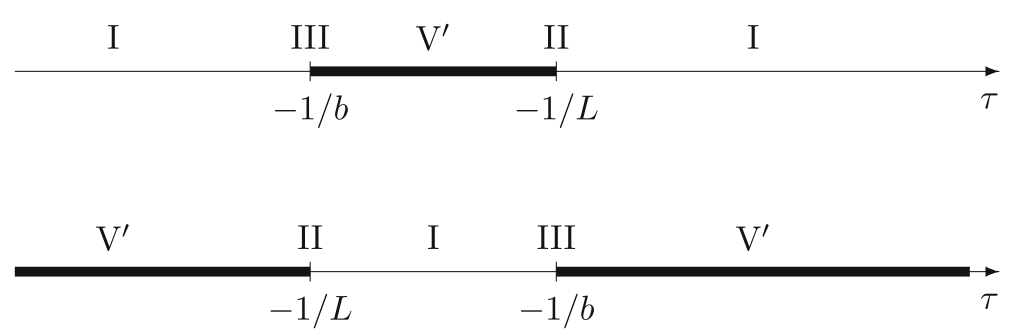

Fig. $1 Q$ in I; $b<L$ have the same signs or opposite signs, respectively

\begin{tabular}{cccccc}
$\mathrm{V}^{\prime}$ & $\mathrm{II}$ & $\mathrm{I}$ & $\mathrm{III}$ & $\mathrm{V}^{\prime}$ & \\
\hline & $-1 / L$ & & $-1 / b$ & & \\
$\mathrm{I}$ & $\stackrel{-1 / b}{\mathrm{III}}$ & $\mathrm{V}^{\prime}$ & $\mathrm{II}$ & $\mathrm{I}$ & \\
\hline & & $-1 / L$ & & $\tau$
\end{tabular}

Fig. $2 Q$ in $\mathrm{V}^{\prime} ; b>L$ have the same signs or opposite signs, respectively

the same sign, then the set $-1 / b<\tau<-1 / L$ is a bounded interval where $b_{\tau}>L_{\tau}$ have opposite signs. If $b<L$ have opposite signs, then the set $-1 / b<\tau<-1 / L$ is the union of two unbounded intervals where $b_{\tau}>L_{\tau}$ have the same sign. The cases $b=0$ and $L=0$ can be seen as limiting cases. The function $Q$ is $\mathrm{Kac}$ at $\infty$ and at 0 ; hence the value $\tau=-1 / b$ is exceptional for the Kac property at $\infty$ and the value $\tau=-1 / L$ is exceptional for the Kac property at 0 .

The situation is completely similar when $Q$ is a Nevanlinna function of type $\mathrm{V}^{\prime}$ (so that $-\infty<L<b<\infty$ ). The behavior of the linear fractional transform $Q_{\tau}$ is sketched in Fig. 2. If $b>L$ have the same sign, then the set $-1 / b<\tau<-1 / L$ is the union of two unbounded intervals where $b_{\tau}<L_{\tau}$ have opposite signs. If $b>L$ have opposite signs, then the set $-1 / b<\tau<-1 / L$ is a bounded interval where $b_{\tau}<L_{\tau}$ have the same sign. Note that if the Nevanlinna function $Q$ is in I and, for instance, $b<L$ have the same signs then for $-1 / b<\tau<-1 / L$, the functions $Q_{\tau}$ belong to $\mathrm{V}^{\prime}$ and $b_{\tau}>L_{\tau}$ have opposite signs; hence the first (second) situation in Fig. 1 corresponds to the second (first) situation in Fig. 2.

Finally let the Nevanlinna function $Q$ belong to the class $\mathrm{V}^{\prime \prime}$. The behavior of the linear fractional transform $Q_{\tau}$ is sketched in Fig. 3. This case can be seen as a limiting case of the earlier situations.

So far the situation has been sketched for Nevanlinna functions of type I and V. If $Q$ is a Nevanlinna function of type II, then $L=\infty$ and $-1 / L=0$. The function $Q$ is Kac at $\infty$ and the value $\tau=-1 / b$ is exceptional for the Kac property at $\infty$. The function $Q$ is not Kac at 0 . Similarly, if $Q$ is a Nevanlinna function of type III, then $b=-\infty$ and $-1 / b=0$. The function $Q$ is Kac at 0 and the value $\tau=-1 / L$ is exceptional for the Kac property at 0 . The function $Q$ is not Kac at $\infty$. If $Q$ is a Nevanlinna function 


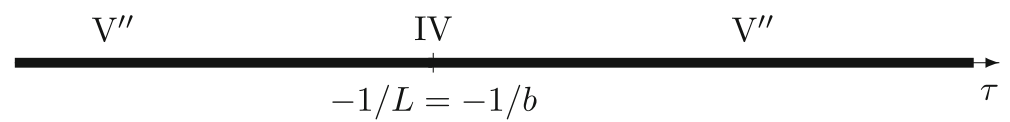

Fig. $3 Q$ in $\mathrm{V}^{\prime \prime}$

of type IV, then $b=-\infty$ and $L=+\infty$, so that $-1 / b=-1 / L=0$. Such a function is neither Kac at $\infty$ nor Kac at 0 .

Note that when starting with a Nevanlinna function $Q$ in I or V one obtains exceptional values of $\tau$ with corresponding functions in II, III, or IV. In the present context it is possible to characterize these exceptional functions.

Corollary 3.8 Let $P$ be a Nevanlinna function in II or III. Then there exists a Nevanlinna function $Q$ in $\mathrm{I}$ or $\mathrm{V}^{\prime}$ such that $P$ is the function corresponding to the exceptional value corresponding to $Q$. If $P$ is a Nevanlinna function in $\mathrm{IV}$, then there exists a Nevanlinna function $Q$ in $\mathrm{V}^{\prime \prime}$ such that $P$ is the function corresponding to the exceptional value corresponding to $Q$.

Proof Let $P$ be a Nevanlinna function of type III, so that $b_{P}=-\infty$ and $L_{P}$ is finite. Then, according to Proposition 3.6, the function $Q$ defined by $Q=P_{\tau_{0}}$ for any $\tau_{0} \in \mathbb{R} \cup\{\infty\}$ with $-1 / \tau_{0}>L_{P}$ is of type I and, clearly,

$$
b_{Q}=\frac{b_{P}-\tau_{0}}{1+\tau_{0} b_{P}}=\frac{1}{\tau_{0}}, \quad L_{Q}=\frac{L_{P}-\tau_{0}}{1+\tau_{0} P} .
$$

Now observe that, conversely, $P$ can be written as a linear fractional transformation of $Q$ ( $Q$ being of type I). In particular, observe that $P=Q_{-\tau_{0}}$, where $-\tau_{0}=-1 / b_{Q}$. The value $-\tau_{0}$ is the exceptional value corresponding to the function $Q$.

\subsection{Some Group Theoretic Aspects of Linear Fractional Transformations} of Nevanlinna Functions

In this subsection some of the facts appearing in the previous subsections are explored by means of the action of the group which generates the linear fractional transforms (3.1) of Nevanlinna functions. ${ }^{1}$ Recall that the automorphism group of the Riemann sphere $\mathbb{S}^{2}$ consists of the Möbius transformations of the form

$$
f(z)=\frac{a z+b}{c z+d}, \quad z \in \mathbb{C} \cup\{\infty\} \cong \mathbb{S}^{2},
$$

where $a, b, c$, and $d$ are complex numbers such that $a d-b c \neq 0$. These transformations are generated by $2 \times 2$ complex matrices

$$
\left(\begin{array}{ll}
a & b \\
c & d
\end{array}\right), \quad a d-b c \neq 0
$$

\footnotetext{
1 The algebraic interpretation of some facts in Sect. 3 on the transforms (3.1) via elements of the automorphism group of the Riemann sphere was suggested by an anonymous referee.
} 
two matrices yield the same Möbius transformation if and only if they differ by a nonzero factor. Clearly, these matrices form a group under matrix multiplication; they are usually called the projective linear transformations and the group is often denoted by $\mathrm{PGL}_{2}(\mathbb{C})$. The linear fractional transforms (3.1) correspond to the subgroup, say $\mathrm{F}$, of $\mathrm{PGL}_{2}(\mathbb{C})$ consisting of complex matrices $\pm F_{\tau}$, where

$$
F_{\tau}=\frac{1}{\sqrt{1+\tau^{2}}}\left(\begin{array}{cc}
1 & -\tau \\
\tau & 1
\end{array}\right), \quad \tau \in \mathbb{R}, \quad(J:=) F_{\infty}=\left(\begin{array}{cc}
0 & -1 \\
1 & 0
\end{array}\right), \quad \tau=\infty .
$$

Note that $F_{\tau}^{-1}=F_{-\tau}$ and that $F_{\sigma} F_{\tau}=\operatorname{sgn}(1-\sigma \tau) F_{\frac{\sigma+\tau}{1-\sigma \tau}}$, if $1-\sigma \tau \neq 0$, and $F_{\sigma} F_{\tau}=\operatorname{sgn}(\tau+1 / \tau) F_{\infty}$, if $1-\sigma \tau=0$; cf. (3.2). In fact, the matrices $\pm F_{\tau}$ are $J$-unitary, i.e., $\left( \pm F_{\tau}\right)^{*} J\left( \pm F_{\tau}\right)=J$, and the corresponding transforms (3.1) map the (closed) upper halfplane into itself (see e.g. [14, Lemma 7.9]); in particular they map $\mathbb{R} \cup\{\infty\} \cong \mathbb{S}^{1}$ bijectively into itself. The identity element of the subgroup corresponds to $\tau=0$, i.e. $F_{0}=I_{2}$, and the formulas for $F_{\sigma} F_{\tau}$ combined with (3.2) mean that the linear fractional transforms (3.1) can be considered as the (left) group action of the subgroup $\mathrm{F}$ of $\mathrm{PGL}_{2}(\mathbb{C})$ on the set of Nevanlinna functions. It is well known that the linear fractional transforms $Q_{\tau}, \tau \in \mathbb{R} \cup\{\infty\}$, of a (nonconstant) Nevanlinna function $Q$ determine all $Q$-functions of a symmetric operator $S$ with defect numbers $(1,1)$; cf. Sect. 5. Using group action terminology they form the orbit of $Q$ under the action of F: $\mathrm{F} Q=\left\{Q_{\tau}: \tau \in \mathbb{R} \cup\{\infty\}\right\}$. The action of the group $\mathrm{F}$ allows some further interpretations and a more structural point of view for some statements given in the previous subsections; these are formulated in the next remark.

Remark 3.9 ${ }^{2}$ Since the transforms (3.1) map (the extended real line) $\mathbb{R} \cup\{\infty\} \cong \mathbb{S}^{1}$ bijectively into itself, it is clear that the collection of Nevanlinna functions $Q$ of type $\mathrm{I}-\mathrm{V}$ is invariant under the action of $\mathrm{F}$; this gives an interpretation and another proof for Lemma 3.5. In fact, these functions can be extended to analytic maps from $\mathbb{C} \backslash[0, \infty)$ to the Riemann sphere $\mathbb{S}^{2}$ with the property that $Q \uparrow(-\infty, 0)$ is injective. The contents of Corollary 3.7 is that under the action of $F$ the collection of Nevanlinna functions of type I-V is divided into two orbits, one of which consists of the types I-III and $\mathrm{V}^{\prime}$, the other one of the types IV and $\mathrm{V}^{\prime \prime}$. These orbits are invariant under the action of F. On each orbit the action of a group is automatically transitive, i.e. for any $Q$ and $\widetilde{Q}$ belonging to the same orbit there exists a transform $F_{\tau} \in \mathrm{F}$ such that $F_{\tau} Q=\widetilde{Q}$. This observation combined with Proposition 3.6 explains from a group action point of view in particular the contents of Corollary 3.8 .

\section{The Exceptional Linear Fractional Transformations}

Let $Q$ be a Nevanlinna function of type I-V with $b=Q(-\infty)$ and $L=Q(0)$, and let $Q_{\tau}, \tau \in \mathbb{R} \cup\{\infty\}$, be the linear fractional transformation defined in (3.1). The aim of this section is to show that the Nevanlinna functions $Q_{\tau}$ of type II, III, and IV which appear in Proposition 3.6 and correspond to the exceptional values $\tau=-1 / b$ and

\footnotetext{
${ }^{2}$ The facts in this remark were communicated to the authors by an anonymous referee.
} 
$\tau=-1 / L$ can be considered as limiting cases of functions $Q_{\tau}$ of type $\mathrm{V}^{\prime}$ and $\mathrm{V}^{\prime \prime}$ as $\tau \downarrow-1 / b$ or $\tau \uparrow-1 / L$, respectively. In particular, it will be shown that in the limit the negative pole $a_{\tau}$ of the approximating functions $Q_{\tau}$ (which tends to $-\infty$ or 0 ) can be interpreted as a point mass or an integrability condition for the measure in the integral representation of the limit function.

The following three cases can occur; cf. Proposition 3.6 and Figs. 1, 2, and 3.

(i) $Q$ is of type I. Then $Q_{\tau}$ is of type III if $\tau=-1 / b$ and $Q_{\tau}$ is of type II if $\tau=-1 / L$. Moreover, $Q_{\tau}$ is of type $\mathrm{V}^{\prime}$ if $b<-1 / \tau<L ;$

(ii) $Q$ is of type $\mathrm{V}^{\prime}$. Then $Q_{\tau}$ is of type III if $\tau=-1 / b$ and $Q_{\tau}$ is of type II if $\tau=-1 / L$. Moreover, $Q_{\tau}$ is of type $\mathrm{V}^{\prime}$ if $-1 / \tau<L$ or $-1 / \tau>b$;

(iii) $Q$ is of type $\mathrm{V}^{\prime \prime}$. Then $Q_{\tau}$ is of type IV if $\tau=-1 / b=-1 / L$, and of type $\mathrm{V}^{\prime \prime}$ otherwise.

Recall that by Proposition 2.6 a Nevanlinna function $Q$ of type I, $\mathrm{V}^{\prime}$, or $\mathrm{V}^{\prime \prime}$ admits the integral representation

$$
Q(\lambda)=Q(-\infty)+\frac{m}{a-\lambda}+\int_{(0, \infty)} \frac{d \sigma(s)}{s-\lambda},
$$

where $Q(-\infty) \in \mathbb{R}, \int_{(0, \infty)} \frac{d \sigma(s)}{s}<\infty$, and $m=0$ if $Q$ is of type I, and $m>0, a<0$, and

$$
Q(-\infty)-Q(0) \begin{cases}>0 & \text { if } Q \text { is of type } \mathrm{V}^{\prime} \\ =0 & \text { if } Q \text { is of type } \mathrm{V}^{\prime \prime}\end{cases}
$$

Observe that for $m=0$ (4.1) reduces to the integral representation in Proposition 2.6 (i), and that in this sense the integral representations of the types $\mathrm{V}^{\prime}$ and $\mathrm{V}^{\prime \prime}$ can be seen as generalizations of type $\mathrm{I}$.

\subsection{The Approximating Functions}

In any of the three cases (i)-(iii) the Nevanlinna functions that correspond to the exceptional values $-1 / b$ and $-1 / L$ will be approximated by functions $Q_{\tau}$ of type $\mathrm{V}$. The functions $Q_{\tau}$ of type $\mathrm{V}$ admit the integral representation

$$
Q_{\tau}(\lambda)=Q_{\tau}(-\infty)+\frac{m_{\tau}}{a_{\tau}-\lambda}+\int_{(0, \infty)} \frac{d \sigma_{\tau}(s)}{s-\lambda}
$$

where $m_{\tau}>0, a_{\tau}<0, \int_{(0, \infty)} \frac{d \sigma_{\tau}(s)}{s}<\infty$, and

$$
Q_{\tau}(-\infty)-Q_{\tau}(0)=-\left(\frac{m_{\tau}}{a_{\tau}}+\int_{(0, \infty)} \frac{d \sigma_{\tau}(s)}{s}\right) \geq 0
$$


cf. Proposition 2.6 (v). The next lemma is essential for the following considerations.

Lemma 4.1 Let $Q$ be a Nevanlinna function as in (i)-(iii) and let $Q_{\tau}$ be of type $\mathrm{V}$ with integral representation (4.2) and (4.3). Then the negative pole $a_{\tau}$ of $Q_{\tau}$ satisfies

$$
\lim _{\tau \downarrow-1 / b} a_{\tau}=-\infty \text { and } \lim _{\tau \uparrow-1 / L} a_{\tau}=0,
$$

and $Q_{\tau}$ admits the integral representation

$$
Q_{\tau}(\lambda)=Q_{\tau}(0)+\frac{m_{\tau}}{\left(a_{\tau}-\lambda\right) a_{\tau}} \lambda+\int_{(0, \infty)}\left(\frac{1}{s-\lambda}-\frac{1}{s}\right) d \sigma_{\tau}(s),
$$

where

$$
m_{\tau}=\frac{\tau^{2}+1}{\tau^{2}} \frac{1}{Q^{\prime}\left(a_{\tau}\right)}
$$

Proof Since the pole $a_{\tau}$ of $Q_{\tau}$ is the unique solution of $Q(\lambda)=-1 / \tau$ on $(-\infty, 0)$, it follows that (4.4) holds. The integral representation (4.5) of $Q_{\tau}$ is a direct consequence of (4.2) and (4.3). Moreover, $-m_{\tau}$ in (4.2) is the corresponding residue of $Q_{\tau}$ at $\lambda=a_{\tau}$; see (2.2). Clearly, in (4.4) one can assume that $\tau \neq 0$ and, therefore,

$$
\begin{aligned}
-m_{\tau} & =\lim _{\lambda \rightarrow a_{\tau}}\left(\lambda-a_{\tau}\right) Q_{\tau}(\lambda)=\lim _{\lambda \rightarrow a_{\tau}}\left(\lambda-a_{\tau}\right) \frac{Q(\lambda)-\tau}{1+\tau Q(\lambda)} \\
& =\lim _{\lambda \rightarrow a_{\tau}} \frac{Q(\lambda)-\tau}{\tau} \frac{\lambda-a_{\tau}}{Q(\lambda)+1 / \tau}=\lim _{\lambda \rightarrow a_{\tau}} \frac{Q(\lambda)-\tau}{\tau} \frac{\lambda-a_{\tau}}{Q(\lambda)-Q\left(a_{\tau}\right)},
\end{aligned}
$$

which leads to (4.6).

\subsection{Cases (i) and (ii) with $\tau=-1 / b$}

Let $Q$ be of type I or type $\mathrm{V}^{\prime}$ with corresponding integral representation (4.1). For convenience the notation $F=-1 / b$ will be used for subscripts. For $\tau \downarrow$ $-1 / b$ the limiting function $Q_{F}=Q_{-1 / b}$ belongs to the class III. According to Proposition 2.6 (iii) the function $Q_{F}$ admits the integral representation

$$
Q_{F}(\lambda)=L_{F}+\beta_{F} \lambda+\int_{(0, \infty)}\left(\frac{1}{s-\lambda}-\frac{1}{s}\right) d \sigma_{F}(s)
$$

where $L_{F}=Q_{F}(0) \in \mathbb{R}, \int_{(0, \infty)} \frac{d \sigma_{F}(s)}{s+s^{2}}<\infty$, and

$$
\beta_{F}>0 \text { or } \int_{(0, \infty)} \frac{d \sigma_{F}(s)}{s+1}=\infty
$$


Observe that the function $Q_{F}$ is exceptional in the sense that for all other values $\tau \in \mathbb{R} \cup\{\infty\}, \tau \neq-1 / b$, one has $b_{\tau}=Q_{\tau}(-\infty) \in \mathbb{R}$, and hence

$$
\lim _{\lambda \rightarrow \infty} \frac{Q_{\tau}(\lambda)}{\lambda}=0 \text { and } \int_{0}^{\infty} \frac{d \sigma_{\tau}(s)}{s+1}<\infty .
$$

From (2.2), (3.1), and the integral representation (4.1) one concludes

$$
\begin{aligned}
\beta_{F} & =\lim _{\lambda \rightarrow \infty} \frac{Q_{F}(\lambda)}{\lambda}=\lim _{\lambda \rightarrow \infty} \frac{b Q(\lambda)+1}{(b-Q(\lambda)) \lambda}=\lim _{\lambda \rightarrow-\infty} \frac{b Q(\lambda)+1}{\frac{\lambda m}{\lambda-a}+\int_{0}^{\infty} \frac{\lambda d \sigma(s)}{\lambda-s}} \\
& =\frac{b^{2}+1}{m+\int_{0}^{\infty} d \sigma(s)},
\end{aligned}
$$

which also shows that the occurence or absence of the linear term in (4.7) is equivalent to

$$
\int_{0}^{\infty} d \sigma(s)<\infty \text { or } \int_{0}^{\infty} d \sigma(s)=\infty
$$

respectively.

The next theorem states that the individual terms in (4.5) converge to the corresponding terms in (4.7). In particular, the linear term in (4.7) can be seen as a limit in the approximation procedure.

Theorem 4.2 Let $Q$ be of type I or type $\mathrm{V}^{\prime}$ with corresponding integral representation (4.1). For $\tau \downarrow-1 / b$ the individual terms in (4.5) converge as follows:

$$
\lim _{\tau \downarrow-1 / b} Q_{\tau}(0)=L_{F} \quad\left(=\frac{1+b L}{b-L} \in \mathbb{R}\right),
$$

and uniformly on compact subsets of $\mathbb{C}_{+}$one has

$$
\lim _{\tau \downarrow-1 / b} \frac{m_{\tau}}{\left(a_{\tau}-\lambda\right) a_{\tau}} \lambda=\beta_{F} \lambda \quad\left(=\frac{b^{2}+1}{m+\int_{0}^{\infty} d \sigma(s)} \lambda\right)
$$

and 


$$
\lim _{\tau \downarrow-1 / b} \int_{(0, \infty)}\left(\frac{1}{s-\lambda}-\frac{1}{s}\right) d \sigma_{\tau}(s)=\int_{(0, \infty)}\left(\frac{1}{s-\lambda}-\frac{1}{s}\right) d \sigma_{F}(s) .
$$

Proof Consider the representation (4.5). The formula (4.10) is obtained directly from the definition (3.1) and (3.5):

$$
\lim _{\tau \rightarrow-1 / b} Q_{\tau}(0)=\lim _{\tau \rightarrow-1 / b} \frac{Q(0)-\tau}{1+\tau Q(0)}=\frac{Q(0)+1 / b}{1-Q(0) / b}=Q_{F}(0)=L_{F} .
$$

Since $b, L=Q(0) \in \mathbb{R}$ and $b<L$ or $L<b$ one has $L_{F} \in \mathbb{R}$. It follows from (4.6) that

$$
\frac{m_{\tau}}{\left(a_{\tau}-\lambda\right) a_{\tau}}=\frac{\tau^{2}+1}{\tau^{2}} \frac{1}{1-\frac{\lambda}{a_{\tau}}} \frac{1}{a_{\tau}^{2} Q^{\prime}\left(a_{\tau}\right)}
$$

holds. Due to (4.4), it follows that $\lambda / a_{\tau} \rightarrow 0$ as $\tau \downarrow-1 / b$ uniformly on compact subsets of $\mathbb{C}_{+}$. The expressions (2.3) and (4.1) for $Q^{\prime}$ and $Q$ are used to determine the following limit:

$$
\begin{aligned}
\lim _{\tau \downarrow-1 / b} a_{\tau}^{2} Q^{\prime}\left(a_{\tau}\right) & =\lim _{\tau \downarrow-1 / b}\left(\frac{m a_{\tau}^{2}}{\left(a-a_{\tau}\right)^{2}}+\int_{0}^{\infty}\left(\frac{a_{\tau}}{s-a_{\tau}}\right)^{2} d \sigma(s)\right) \\
& =m+\int_{0}^{\infty} d \sigma(s),
\end{aligned}
$$

where the monotone convergence theorem has been used. Hence, in the limit one obtains

$$
\lim _{\tau \downarrow-1 / b} \frac{m_{\tau}}{\left(a_{\tau}-\lambda\right) a_{\tau}}=\frac{b^{2}+1}{m+\int_{0}^{\infty} d \sigma(s)}
$$

uniformly on compact subsets of $\mathbb{C}_{+}$, and since the coefficient $\beta_{F}$ in (4.7) is given by (4.9), the statement in (4.11) follows. Finally, as $Q_{\tau} \rightarrow Q_{F}$ uniformly on compact subsets of $\mathbb{C}_{+}$for $\tau \downarrow-1 / b$ by Lemma 3.1, the formula (4.12) follows from (4.10) and (4.11).

The interpretation of the limit relation (4.11) is that for $\tau \downarrow-1 / b$ the jump of $\sigma_{\tau}$ at $a_{\tau}$ produces the linear term in (4.7) if $\beta_{F}>0$ (or, equivalently, $\int_{0}^{\infty} d \sigma(s)<\infty$ ) or leads to the condition

$$
\int_{(0, \infty)} \frac{d \sigma_{F}(s)}{s+1}=\infty
$$

cf. (4.8). 
4.3 Cases (i) and (ii) with $\tau=-1 / L$

Let $Q$ be of type I or type $\mathrm{V}^{\prime}$ with corresponding integral representation (4.1). For convenience the notation $N=-1 / L$ will be used for subscripts. For $\tau \downarrow$ $-1 / L$ the limiting function $Q_{N}=Q_{-1 / L}$ belongs to the class II. According to Proposition 2.6 (ii) the function $Q_{N}$ admits the integral representation

$$
Q_{N}(\lambda)=b_{N}+\int_{[0, \infty)} \frac{d \sigma_{N}(s)}{s-\lambda}
$$

where

$$
b_{N} \in \mathbb{R}, \quad \int_{[0, \infty)} \frac{d \sigma_{N}(s)}{1+s}<\infty, \int_{[0,1]} \frac{d \sigma_{N}(s)}{s}=\infty
$$

see Proposition 2.6 (ii). Note that $Q_{N}$ is exceptional in the sense that for all other values $\tau \in \mathbb{R} \cup\{\infty\}, \tau \neq-1 / L$, one has $L_{\tau}=Q_{\tau}(0) \in \mathbb{R}$, and hence

$$
\int_{[0,1]} \frac{d \sigma_{\tau}(s)}{s}<\infty
$$

Observe, that if $Q$ has the integral representation (4.1), then (cf. (4.5)):

$$
\begin{aligned}
\lim _{\lambda \rightarrow 0} \frac{Q(\lambda)-Q(0)}{\lambda} & =\lim _{\lambda \uparrow 0}\left(\frac{m}{(a-\lambda) a}+\int_{(0, \infty)} \frac{d \sigma(s)}{s(s-\lambda)}\right) \\
& =\frac{m}{a^{2}}+\int_{(0, \infty)} \frac{d \sigma(s)}{s^{2}} \leq \infty,
\end{aligned}
$$

where the monotone convergence theorem has been used. This implies that

$$
\lim _{\lambda \rightarrow 0}(0-\lambda) Q_{N}(\lambda)=\lim _{\lambda \rightarrow 0} \frac{-L Q(\lambda)-1}{(L-Q(\lambda)) / \lambda}=\frac{L^{2}+1}{m / a^{2}+\int_{(0, \infty)} d \sigma(s) / s^{2}},
$$

which shows that the occurrence or absence of a jump of $\sigma_{N}$ at $s=0$ in (4.13) is equivalent to

$$
\int_{[0, \infty)} d \sigma(s) / s^{2}<\infty \text { or } \int_{[0, \infty)} d \sigma(s) / s^{2}=\infty
$$

respectively; cf. (2.2).

Next it will be shown that the individual terms in the integral representation (4.2) of $Q_{\tau}$ converge to the corresponding terms in (4.13). 
Theorem 4.3 Let $Q$ be of type I or type $\mathrm{V}^{\prime}$ with corresponding integral representation (4.1). For $\tau \uparrow-1 / L$ the individual terms in (4.2) converge as follows:

$$
\lim _{\tau \uparrow-1 / L} Q_{\tau}(-\infty)=b_{N} \quad\left(=\frac{1+b L}{L-b} \in \mathbb{R}\right)
$$

and uniformly on compact subsets of $\mathbb{C}_{+}$one has

$$
\lim _{\tau \uparrow-1 / L} \frac{m_{\tau}}{a_{\tau}-\lambda}=\frac{L^{2}+1}{\frac{m}{a^{2}}+\int_{0}^{\infty} \frac{d \sigma(s)}{s^{2}}}\left(-\frac{1}{\lambda}\right)=\frac{\sigma_{N}(0+)-\sigma_{N}(0-)}{-\lambda}
$$

and

$$
\lim _{\tau \uparrow-1 / L} \int_{(0, \infty)} \frac{d \sigma_{\tau}(s)}{s-\lambda}=\int_{(0, \infty)} \frac{d \sigma_{N}(s)}{s-\lambda} .
$$

Proof Consider the representation of $Q_{\tau}$ in (4.2) and (4.3). The formula (4.16) is obtained from the definitions in (3.1) and (3.5):

$\lim _{\tau \uparrow-1 / L} Q_{\tau}(-\infty)=\lim _{\tau \uparrow-1 / L} \frac{Q(-\infty)-\tau}{1+\tau Q(-\infty)}=\frac{Q(-\infty)+1 / L}{1-Q(-\infty) / L}=Q_{N}(-\infty)=b_{N}$

Since $b=Q(-\infty), L \in \mathbb{R}$, and $b<L$ or $L<b$ one has $b_{N} \in \mathbb{R}$.

It follows from (4.6) that

$$
\frac{m_{\tau}}{a_{\tau}-\lambda}=\frac{\tau^{2}+1}{\tau^{2}} \frac{1}{Q^{\prime}\left(a_{\tau}\right)} \frac{1}{a_{\tau}-\lambda}
$$

holds. Due to (4.4), $a_{\tau}-\lambda \rightarrow-\lambda$ as $\tau \uparrow-1 / L$ uniformly on compact subsets of $\mathbb{C}_{+}$. The expressions (2.3) and (4.1) for $Q^{\prime}$ and $Q$ yield

$$
\begin{aligned}
\lim _{\tau \uparrow-1 / L} Q^{\prime}\left(a_{\tau}\right) & =\left(\lim _{\tau \rightarrow-1 / L} \frac{m}{\left(a-a_{\tau}\right)^{2}}+\int_{0}^{\infty}\left(\frac{1}{s-a_{\tau}}\right)^{2} d \sigma(s)\right) \\
& =\frac{m}{a^{2}}+\int_{0}^{\infty} \frac{d \sigma(s)}{s^{2}},
\end{aligned}
$$

where the monotone convergence theorem has been used. Hence in the limit one obtains the first equality in (4.17) uniformly on compact subsets of $\mathbb{C}_{+}$and the second equality follows from (2.2) and (4.15). Since $Q_{\tau} \rightarrow Q_{N}$ uniformly on compact subsets of $\mathbb{C}_{+}$as $\tau \uparrow-1 / L$ by Lemma 3.1, the formula (4.18) follows from (4.16) and (4.17). 
The interpretation of (4.17) and (4.18) is that the jump of at $a_{\tau}<0$ produces the possible jump of $\sigma_{N}$ at 0 , see (4.15) and the discussion after it. In fact, as is usual, the jump of $\sigma_{N}$ at 0 can be included in (4.18) and by combining the term $m_{\tau} /\left(a_{\tau}-\lambda\right)$ with the integral term of $Q_{\tau}$ in (4.2) one concludes that together (4.17) and (4.18), show that (for all $a_{\tau}>-1$ ), uniformly on compact subsets,

$$
\lim _{\tau \uparrow-1 / L} \int_{-1}^{\infty} \frac{d \sigma_{\tau}(s)}{s-\lambda}=\int_{[0, \infty)} \frac{d \sigma_{N}(s)}{s-\lambda} .
$$

\subsection{Case (iii)}

Let $Q$ be of type $\mathrm{V}^{\prime \prime}$ with corresponding integral representation (4.1). As $b=L$ also $F=-1 / b=-1 / L=N$ and $Q_{F}=Q_{N}$ belongs to the class IV. According to Proposition 2.6 (iv) this function admits the integral representation

$$
Q_{F}(\lambda)=\beta_{F} \lambda+\alpha_{F}+\int_{[0, \infty)}\left(\frac{1}{s-\lambda}-\frac{s}{s^{2}+1}\right) d \sigma_{F}(s)
$$

where $\int_{[0, \infty)} \frac{d \sigma_{F}(s)}{1+s^{2}}<\infty, \int_{[0, \infty)} \frac{d \sigma_{F}(s)}{s(1+s)}=\infty$, and

$$
\beta_{F}>0 \text { or } \int_{[0, \infty)} \frac{d \sigma_{F}(s)}{1+s}=\infty
$$

The following variant of Theorems 4.2 and 4.3 holds for the convergence of the individual terms in (4.5) and (4.2) to the corresponding terms in (4.19). The proof is essentially the same as the proof of Theorems 4.2 and 4.3 and is therefore not repeated.

Theorem 4.4 Let $Q$ be of type $\mathrm{V}^{\prime \prime}$ with corresponding integral representation (4.1) and let $Q_{F}=Q_{N}$ be as in (4.19). Then the following statements hold:

(F) For $\tau \downarrow-1 / b=-1 / L$ the individual terms in (4.5) converge as follows:

$$
\lim _{\tau \downarrow-1 / b} Q_{\tau}(0)=+\infty
$$

and uniformly on compact subsets of $\mathbb{C}_{+}$one has

$$
\lim _{\tau \downarrow-1 / b} \frac{m_{\tau}}{\left(a_{\tau}-\lambda\right) a_{\tau}} \lambda=\beta_{F} \lambda\left(=\frac{b^{2}+1}{m+\int_{0}^{\infty} d \sigma(s)} \lambda\right)
$$

and

$$
\lim _{\tau \downarrow-1 / b}\left(Q_{\tau}(0)+\int_{(0, \infty)}\left(\frac{1}{s-\lambda}-\frac{1}{s}\right) d \sigma_{\tau}(s)\right)=Q_{F}(\lambda)-\beta_{F} \lambda .
$$


(N) For $\tau \uparrow-1 / L=-1 / b$ the individual terms in (4.2) converge as follows:

$$
\lim _{\tau \uparrow-1 / L} Q_{\tau}(-\infty)=-\infty
$$

and uniformly on compact subsets of $\mathbb{C}_{+}$one has

$$
\lim _{\tau \uparrow-1 / L} \frac{m_{\tau}}{a_{\tau}-\lambda}=\frac{L^{2}+1}{\frac{m}{a^{2}}+\int_{0}^{\infty} \frac{d \sigma(s)}{s^{2}}}\left(-\frac{1}{\lambda}\right)=\frac{\sigma_{N}(0+)-\sigma_{N}(0-)}{-\lambda}
$$

and

$$
\lim _{\tau \uparrow-1 / L}\left(Q_{\tau}(-\infty)+\int_{(0, \infty)} \frac{d \sigma_{\tau}(s)}{s-\lambda}\right)=Q_{N}(\lambda)+\frac{\sigma_{N}(0+)-\sigma_{N}(0-)}{\lambda} .
$$

\section{Nonnegative Symmetric Operators and Their Weyl Functions}

The aim of this section is to explain and interpret the function-theoretic results in the previous sections in an operator-theoretical framework. It will be shown that the Weyl function of a nonnegative symmetric operator $S$ with defect numbers $(1,1)$ is a Nevanlinna function of type $\mathrm{I}-\mathrm{V}$, the selfadjoint extensions $A_{\tau}$ of $S$ and their possible negative eigenvalue will be described, and the connection of the exceptional values $\tau=-1 / b$ and $\tau=-1 / L$ with the Friedrichs and Krel̆n-von Neumann extension of $S$ is explained.

\subsection{Boundary Triplets, Weyl Functions, and Linear Fractional Transformations}

The notions of boundary triplets and Weyl functions are efficient tools for the description of the closed extensions of a symmetric operator or relation with equal defect numbers. The reader is referred to, e.g., [4,5] for a detailed investigation of boundary triplets and various applications in the extension theory of symmetric operators and relations. In the following let $S$ be a closed symmetric (not necessarily densely defined) operator with defect numbers $(1,1)$ in a Hilbert space $(\mathfrak{H},(\cdot, \cdot))$. The adjoint $S^{*}$ is an operator if and only if the domain of $S$ is dense in $\mathfrak{H}$, and a linear relation with a one-dimensional multivalued part otherwise. For more details on linear relations the reader is refered to [13].

Definition 5.1 A boundary triplet $\left\{\mathbb{C}, \Gamma_{0}, \Gamma_{1}\right\}$ for $S^{*}$ consists of two linear functionals $\Gamma_{0}$ and $\Gamma_{1}$ defined on $S^{*}$ such that $\left(\Gamma_{0}, \Gamma_{1}\right)^{\top}: S^{*} \rightarrow \mathbb{C}^{2}$ is onto and the abstract Lagrange or Green's identity

$$
\left(f^{\prime}, g\right)-\left(f, g^{\prime}\right)=\Gamma_{1} \hat{f} \overline{\Gamma_{0} \hat{g}}-\Gamma_{0} \hat{f} \overline{\Gamma_{1} \hat{g}}
$$

holds for all $\hat{f}=\left\{f, f^{\prime}\right\}, \hat{g}=\left\{g, g^{\prime}\right\} \in S^{*}$. 
Assume that $\left\{\mathbb{C}, \Gamma_{0}, \Gamma_{1}\right\}$ is a boundary triplet for $S^{*}$. Then the family of selfadjoint extensions of $S$ in $\mathfrak{H}$ can be parameterized via

$$
A_{\tau}=\operatorname{ker}\left(\Gamma_{0}+\tau \Gamma_{1}\right), \quad \tau \in \mathbb{R} \cup\{\infty\},
$$

with the interpretation $A_{\infty}=\operatorname{ker} \Gamma_{1}$. Recall that if the symmetric operator $S$ is densely defined, then all selfadjoint extensions $A_{\tau}$ are densely defined operators. However, if the operator $S$ is not densely defined then for all but one $\tau \in \mathbb{R} \cup\{\infty\}$ the selfadjoint extensions $A_{\tau}$ are densely defined and for precisely one $\tau \in \mathbb{R} \cup\{\infty\}$ the selfadjoint extension $A_{\tau}$ is a relation with a nontrivial multivalued part $(\operatorname{dom} S)^{\perp}$. Note that in the case when $S$ is a closed symmetric relation one may orthogonally split off the selfadjoint purely-multivalued part of $S$ to reduce to the case of a closed symmetric operator.

In the following the restriction $A_{0}=$ ker $\Gamma_{0}$ will be regarded as a fixed selfadjoint extension of $S$. For $\lambda \in \mathbb{C}$ set

$$
\widehat{\mathfrak{N}}_{\lambda}\left(S^{*}\right)=\left\{\left\{f_{\lambda}, \lambda f_{\lambda}\right\}: f_{\lambda} \in \operatorname{ker}\left(S^{*}-\lambda\right)\right\}
$$

The Weyl function $Q$ (or $Q$-function) associated to the boundary triplet $\left\{\mathbb{C}, \Gamma_{0}, \Gamma_{1}\right\}$ is defined on the resolvent set $\rho\left(A_{0}\right)$ of the selfadjoint operator $A_{0}$ by

$$
\rho\left(A_{0}\right) \ni \lambda \mapsto Q(\lambda)=\frac{\Gamma_{1} \hat{f}_{\lambda}}{\Gamma_{0} \hat{f}_{\lambda}}, \quad \hat{f}_{\lambda}=\left\{f_{\lambda}, \lambda f_{\lambda}\right\} \in \widehat{\mathfrak{N}}_{\lambda}\left(S^{*}\right)
$$

The Weyl function $Q$ is nonconstant, holomorphic on $\rho\left(A_{0}\right)$, and belongs to the Nevanlinna class $\mathbf{N}$.

The study of all selfadjoint extensions $A_{\tau}$ of $S$ can be carried out by describing boundary triplets $\left\{\mathbb{C}, \Gamma_{0}^{\tau}, \Gamma_{1}^{\tau}\right\}$ for $S^{*}$ such that $A_{\tau}=\operatorname{ker} \Gamma_{0}^{\tau}$. In the next proposition a boundary triplet for $S^{*}$ is specified in such a way that the corresponding Weyl function coincides with the linear fractional transformation (3.1). The proof is based on the description of all boundary triplets associated with $S^{*}$ via $J$-unitary operators in (3.6); for a complete proof of the next statement, see for instance [3].

Proposition 5.2 Let $\left\{\mathbb{C}, \Gamma_{0}, \Gamma_{1}\right\}$ be a boundary triplet for $S^{*}$ and let $Q$ be the corresponding Weyl function. Then $\left\{\mathbb{C}, \Gamma_{0}^{\tau}, \Gamma_{1}^{\tau}\right\}$, where

$$
\Gamma_{0}^{\tau}=\frac{1}{\sqrt{1+\tau^{2}}}\left(\Gamma_{0}+\tau \Gamma_{1}\right) \quad \text { and } \quad \Gamma_{1}^{\tau}=\frac{1}{\sqrt{1+\tau^{2}}}\left(\Gamma_{1}-\tau \Gamma_{0}\right), \quad \tau \in \mathbb{R}
$$

is a boundary triplet for $S^{*}$ with $A_{\tau}=\operatorname{ker} \Gamma_{0}^{\tau}$ and corresponding Weyl function

$$
Q_{\tau}(\lambda)=\frac{Q(\lambda)-\tau}{1+\tau Q(\lambda)}, \quad \lambda \in \rho\left(A_{0}\right) \cap \rho\left(A_{\tau}\right)
$$

Moreover, $\left\{\mathbb{C}, \Gamma_{0}^{\infty}, \Gamma_{1}^{\infty}\right\}$, where $\Gamma_{0}^{\infty}=\Gamma_{1}$ and $\Gamma_{1}^{\infty}=-\Gamma_{0}$ is a boundary triplet for $S^{*}$ with corresponding Weyl function $Q_{\infty}(\lambda)=-Q(\lambda)^{-1}, \lambda \in \rho\left(A_{0}\right) \cap \rho\left(A_{\infty}\right)$. 
For spectral analysis, it is convenient to recall Kreĭn's resolvent formula, which connects the resolvents of $A_{0}$ and $A_{\tau}$ via

$$
\left(A_{\tau}-\lambda\right)^{-1}=\left(A_{0}-\lambda\right)^{-1}-\gamma(\lambda)(Q(\lambda)+1 / \tau)^{-1} \gamma(\bar{\lambda})^{*}
$$

for all $\lambda \in \rho\left(A_{0}\right) \cap \rho\left(A_{\tau}\right)$. Here $Q$ is the Weyl function and the vector valued mapping $\gamma(\lambda): \mathbb{C} \rightarrow \mathfrak{H}$, the so-called $\gamma$-field, is defined by

$$
\gamma(\lambda):=\pi_{1}\left(\Gamma_{0}\left\lceil\widehat{\mathfrak{N}}_{\lambda}\left(S^{*}\right)\right)^{-1}, \lambda \in \rho\left(A_{0}\right)\right.
$$

where $\pi_{1}$ denotes the projection onto the first component of $\mathfrak{H} \times \mathfrak{H}$. It follows from Krĕn's resolvent formula that a point $\lambda \in \rho\left(A_{0}\right)$ is an eigenvalue of $A_{\tau}$ if and only if $Q(\lambda)=-1 / \tau$.

\subsection{Weyl Functions for Nonnegative Operators}

In this section it will be assumed that the closed symmetric (not necessarily densely defined) operator $S$ with defect numbers $(1,1)$ is nonnegative. Recall that such an operator always admits (at least one) nonnegative selfadjoint extension which may be multivalued, see also Sect. 5.3. The next proposition shows that in this situation the corresponding Weyl functions are Nevanlinna functions of type I-V.

Proposition 5.3 Let $S$ be a nonnegative operator in a Hilbert space $\mathfrak{H}$ with defect numbers $(1,1)$. Let $\left\{\mathbb{C}, \Gamma_{0}, \Gamma_{1}\right\}$ be a boundary triplet for $S^{*}$ with corresponding Weyl function $Q$. Then either $A_{0}=\operatorname{ker} \Gamma_{0}$ is nonnegative and $Q$ is of type I-IV or the set $\sigma\left(A_{0}\right) \cap(-\infty, 0)$ consists of one eigenvalue with multiplicity one and $Q$ is of type $\mathrm{V}$.

Proof If $A_{0}$ is nonnegative, then the Weyl function $Q$ is a Nevanlinna function which is holomorphic on $(-\infty, 0)$. Therefore $Q$ is of type I-IV in this situation.

If $A_{0}$ is not nonnegative, then choose a nonnegative selfadjoint extension $B$ of $S$. According to Kreŭn's formula (5.3) $A_{0}$ can be written as a one-dimensional perturbation in resolvent sense of $B$. Hence $\sigma\left(A_{0}\right) \cap(-\infty, 0)$ consists of one negative eigenvalue $a$ of multiplicity one and it follows from (5.3) that $a$ is a pole of $Q$. It remains to verify that the injectivity condition in the definition of class $\mathrm{V}$ is satisfied. Suppose this is not the case. Then there exists $c \in \mathbb{R}$ such that the function $Q+c$ has two negative zeros, cf. Lemma 2.5. Since the function $-(Q+c)^{-1}$ is the Weyl function corresponding to the boundary triplet $\left\{\mathbb{C}, \Gamma_{1}+c \Gamma_{0},-\Gamma_{0}\right\}$ for $S^{*}$ this yields that the selfadjoint extension $D:=\operatorname{ker}\left(\Gamma_{1}+c \Gamma_{0}\right)$ has two negative eigenvalues. On the other hand, $D$ is a one dimensional perturbation in resolvent sense of the nonnegative operator $B$, hence $\sigma(D) \cap(-\infty, 0)$ consists of at most one negative eigenvalue of multiplicity one. This contradiction shows that $Q$ belongs to $\mathrm{V}$.

The following corollary is an immediate consequence of Propositions 3.6 and 5.2. Recall that for a Nevanlinna function of type I-V the $\operatorname{limits}_{\lambda \uparrow 0} Q(\lambda)$ and $\lim _{\lambda \downarrow-\infty} Q(\lambda)$ are denoted by $L$ and $b$, respectively; cf. (3.3).

Corollary 5.4 Let $S$ be a nonnegative operator in a Hilbert space $\mathfrak{H}$ with defect numbers $(1,1)$. 
Let $\left\{\mathbb{C}, \Gamma_{0}, \Gamma_{1}\right\}$ be a boundary triplet for $S^{*}$ and let $Q$ be the corresponding Weyl function. Then the following statements holds for the selfadjoint extension $A_{\tau}=$ $\operatorname{ker}\left(\Gamma_{0}+\tau \Gamma_{1}\right), \tau \in \mathbb{R} \cup\{\infty\}$ :

(i) If $Q$ is of type I-III, then

- $A_{\tau}$ is nonnegative for $-1 / \tau \notin(b, L)$;

- $A_{\tau}$ has one negative eigenvalue for $-1 / \tau \in(b, L)$.

(ii) If $Q$ is of type IV, then

- $A_{\tau}$ is nonnegative for $\tau=0$;

- $A_{\tau}$ has one negative eigenvalue for $\tau \neq 0$.

(i') If $Q$ is of type $\mathrm{V}^{\prime}$, then

- $A_{\tau}$ is nonnegative for $\tau \in[-1 / L,-1 / b]$;

- $A_{\tau}$ has one negative eigenvalue for $\tau \notin[-1 / L,-1 / b]$.

(ii') If $Q$ is of type $\mathrm{V}^{\prime \prime}$, then

- $A_{\tau}$ is nonnegative for $\tau=-1 / L=-1 / b$;

- $A_{\tau}$ has one negative eigenvalue for $\tau \neq-1 / L=-1 / b$.

In the cases where $A_{\tau}$ has a negative eigenvalue $a_{\tau}$, this eigenvalue is of multiplicity one and its location is determined by $Q\left(a_{\tau}\right)=-1 / \tau$.

Each nonconstant Nevanlinna function is the Weyl function of a closed symmetric operator $S$. If the Nevanlinna function is holomorphic on $(-\infty, 0)$, then the operator $S$ is clearly nonnegative. Now it follows from Proposition 3.6 that each function of type $\mathrm{I}-\mathrm{V}$ is the Weyl function of some nonnegative operator.

\subsection{Friedrichs and Krĕ̌n-von Neumann Extension of a Nonnegative Operator}

According to Kreĭn [22] a closed symmetric nonnegative operator $S$ admits two nonnegative selfadjoint extensions $A_{F}$ (the Friedrichs extensions) and $A_{N}$ (the Kreĭn-von Neumann extension) such that any nonnegative selfadjoint extension $A_{\tau}$ in $\mathfrak{H}$ of $S$ satisfies

$$
\left(A_{F}-\lambda\right)^{-1} \leq\left(A_{\tau}-\lambda\right)^{-1} \leq\left(A_{N}-\lambda\right)^{-1}, \quad \lambda<0,
$$

and, conversely, if $A_{\tau}$ is a selfadjoint operator whose resolvent satisfies (5.4) then $A_{\tau}$ is a nonnegative selfadjoint extension of $S$; cf. [11]. In this sense, the resolvents of all nonnegative selfadjoint extensions of $S$ form an operator interval

$$
\left[\left(A_{F}-\lambda\right)^{-1},\left(A_{N}-\lambda\right)^{-1}\right], \quad \lambda<0 .
$$

This interval can collapse to a single point, i.e. $\left(A_{F}-\lambda\right)^{-1}=\left(A_{N}-\lambda\right)^{-1}$, which means that there is only one nonnegative selfadjoint extension, namely $A_{N}=A_{F}$.

Assume now, as in the previous subsection, that $S$ is a closed symmetric (not necessarily densely defined) nonnegative operator with defect numbers $(1,1)$ and let $Q$ be a Weyl function. Recall that if $S$ is not densely defined, then the Friedrichs extension is the only selfadjoint extension of $S$ which is not an operator. The next corollary shows that the exceptional values $\tau=-1 / b$ and $\tau=-1 / L$ correspond to the Friedrichs and 
Kreı̆n-von Neumann extension; cf. [5, Proposition 4.2]. A short direct proof based on (5.3) and Corollary 5.4 is included.

Corollary 5.5 Let $\left\{\mathbb{C}, \Gamma_{0}, \Gamma_{1}\right\}$ be a boundary triplet for $S^{*}$ and let $Q$ be the corresponding Weyl function. Then

$$
A_{F}=A_{-1 / b} \quad \text { and } \quad A_{N}=A_{-1 / L} .
$$

Proof Assume that $Q$ is of type I-III. If $Q$ is of type IV or V a similar reasoning applies. In order to prove that $A_{-1 / b}$ coincides with the Friedrich extension $A_{F}$ observe that for $\lambda \in \rho\left(A_{0}\right) \cap \rho\left(A_{\tau}\right) \cap \rho\left(A_{-1 / b}\right) \cap \mathbb{R}$ the resolvent formula (5.3) implies

$$
\left(A_{\tau}-\lambda\right)^{-1}-\left(A_{-1 / b}-\lambda\right)^{-1}=\gamma(\lambda) \widetilde{Q}(\lambda) \gamma(\lambda)^{*},
$$

where

$$
\widetilde{Q}(\lambda)=\frac{1}{Q(\lambda)-b}-\frac{1}{Q(\lambda)+1 / \tau} .
$$

Suppose now that $A_{\tau}$ is a nonnegative selfadjoint extension of $S$. Then by Corollary 5.4 (i) either $-1 / \tau \leq b$ or $-1 / \tau \geq L$. For $\lambda \in(-\infty, 0)$ it follows from $b<Q(\lambda)<L$ that

$$
0<Q(\lambda)-b \leq Q(\lambda)+1 / \tau, \quad \text { if }-1 / \tau \leq b
$$

and

$$
Q(\lambda)+1 / \tau \leq Q(\lambda)-L<0, \quad \text { if }-1 / \tau \geq L,
$$

hold. Therefore, the function $\widetilde{Q}$ in (5.6) is nonnegative on $(-\infty, 0)$. Together with (5.5) this implies

$$
\left(A_{-1 / b}-\lambda\right)^{-1} \leq\left(A_{\tau}-\lambda\right)^{-1}
$$

for all $\lambda \in \rho\left(A_{0}\right) \cap \rho\left(A_{\tau}\right) \cap \rho\left(A_{-1 / b}\right) \cap \mathbb{R}$, where $A_{\tau}$ is an arbitrary nonnegative selfadjoint extension of $S$. This proves $A_{F}=A_{-1 / b}$. An analogous argument can be used to show $A_{N}=A_{-1 / L}$.

Corollary 5.6 Let $\left\{\mathbb{C}, \Gamma_{0}, \Gamma_{1}\right\}$ be a boundary triplet for $S^{*}$ and let $Q$ be the corresponding Weyl function. Then $A_{F}=A_{N}$ if and only if $Q$ is of type IV or $V^{\prime \prime}$.

Note also that if $Q$ in Corollary 5.6 is of type IV, then $A_{F}=A_{N}=A_{0}$, and if $Q$ is of type $\mathrm{V}^{\prime \prime}$, then $A_{F}=A_{N}=A_{-1 / b}=A_{-1 / L}$.

The following simple model example illustrates the situation in Corollary 5.6. 
Example 5.7 The Nevanlinna function $Q(\lambda)=\lambda-1 / \lambda$ is of type IV; it has poles at 0 and $\infty$. The underlying (minimal) symmetric operator $S$ acts in $\mathbb{C}^{2}$ and the corresponding selfadjoint extension $A_{0}$ of $S$ has eigenvalues at 0 and $\infty$, i.e., $A_{0}$ is a selfadjoint relation extension of $S$ given by

$$
A_{0}=\{\{\phi, 0\} \oplus\{0, \psi\}: \phi, \psi \in \mathbb{C}\}
$$

In this case $A_{0}$ is nonnegative, and it is the unique nonnegative selfadjoint extension of $S$, i.e., $A_{0}=A_{F}=A_{N}$. By Theorem 5.4 all the other selfadjoint extensions $A_{\tau}$ of $S$ have one negative eigenvalue.

Let $S$ be a nonnegative operator in a Hilbert space $\mathfrak{H}$ and let the eigenspace $\widehat{\mathfrak{N}}_{\lambda}\left(S^{*}\right), \lambda \in \mathbb{C}$, be defined as in (5.1). Then it is well-known that

$$
A(x)=S \widehat{+} \widehat{\mathfrak{N}}_{x}\left(S^{*}\right), \quad x<0,
$$

is a selfadjoint extension of $S$; here $\widehat{+}$ denotes a componentwise sum (i.e. linear span) of the corresponding graphs.

Assume in addition that $S$ has defect numbers $(1,1)$ and let $\left\{\mathbb{C}, \Gamma_{0}, \Gamma_{1}\right\}$ be a boundary triplet for $S^{*}$ with corresponding Weyl function $Q$. The selfadjoint extensions in (5.7) can now be parametrized in the following way.

Lemma 5.8 For $\tau(x)=-1 / Q(x), x<0$, one has

$$
A(x)=A_{\tau(x)}=\operatorname{ker}\left(\Gamma_{0}+\tau(x) \Gamma_{1}\right) .
$$

Proof With $\tau(x)=-1 / Q(x)$ it follows directly from (5.2) that

$$
A(x)=S \widehat{+} \widehat{\mathfrak{N}}_{x}\left(S^{*}\right) \subset \operatorname{ker}\left(\Gamma_{0}+\tau(x) \Gamma_{1}\right) .
$$

Since $A(x)$ and ker $\left(\Gamma_{0}+\tau(x) \Gamma_{1}\right)$ are both selfadjoint extensions of $S$, it follows that (5.8) holds for all $x<0$.

From the form of this parametrization it is clear that

$$
\tau(x) \downarrow-1 / b \Leftrightarrow x \downarrow-\infty \text { and } \tau(x) \uparrow-1 / L \Leftrightarrow x \uparrow 0 .
$$

In terms of the boundary triplet $\left\{\mathbb{C}, \Gamma_{0}, \Gamma_{1}\right\}$ the Weyl function for the selfadjoint extension $A(x)$ is given by the linear fractional transformation $Q_{\tau(x)}$ :

$$
Q_{\tau(x)}(\lambda)=\frac{Q(\lambda)-\tau(x)}{1+\tau(x) Q(\lambda)}=\frac{1+Q(\lambda) Q(x)}{Q(x)-Q(\lambda)},
$$

with a pole for $\lambda=x(<0)$. If $Q$ is of type $\mathrm{I}$ or $\mathrm{V}^{\prime}$ then Theorems 4.2 and 4.3 show the convergence of the individual terms in the integral representation of $Q_{\tau(x)}$ for $\tau(x) \downarrow-1 / b$ (that is, $x \downarrow-\infty$ ) and $\tau(x) \uparrow-1 / L$ (that is, $x \uparrow 0$ ). If $Q$ is of type $\mathrm{V}^{\prime \prime}$ then Theorem 4.4 applies. 
The following result in the case that the Friedrichs and the Kreĭn-von Neumann extensions exist as densely defined selfadjoint operators goes back to [1]; see also $[2,5,11]$. Proposition 5.9 is the operator-theoretic counterpart to the results in Section 4, where the convergence of the associated Weyl functions $Q_{\tau}$ is described with a pole on $(-\infty, 0)$ moving to the endpoints of this interval.

Proposition 5.9 Let $S$ be a closed, not necessarily densely defined, nonnegative operator in a Hilbert space $\mathfrak{H}$ with defect numbers $(1,1)$. The strong resolvent limits of the selfadjoint extensions $A(x)$ in (5.7) as $x \uparrow 0$ and $x \downarrow-\infty$ are the Krĕ̌n-von Neumann extension $A_{N}$ and the Friedrichs extension $A_{F}$ of $S$, respectively:

$$
\begin{aligned}
& \left(A_{N}-\lambda\right)^{-1} h=\lim _{x \uparrow 0}(A(x)-\lambda)^{-1} h, \\
& \left(A_{F}-\lambda\right)^{-1} h=\lim _{x \downarrow-\infty}(A(x)-\lambda)^{-1} h, \quad \text { for allh } \in \mathfrak{H} .
\end{aligned}
$$

Note that the difference of the resolvents of $A_{N}$ and $A(x)$, and $A_{F}$ and $A(x)$ can be expressed via KreĬn's resolvent formula (5.3) and the functions $Q_{N}$ and $Q_{\tau(x)}$, and $Q_{F}$ and $Q_{\tau(x)}$, respectively. Hence the convergence of the resolvents in Proposition 5.9 also yields convergence results for $Q_{\tau(x)}$ to $Q_{N}$ and $Q_{F}$ for $x \uparrow 0$ and $x \downarrow-\infty$, respectively.

Open Access This article is distributed under the terms of the Creative Commons Attribution Noncommercial License which permits any noncommercial use, distribution, and reproduction in any medium, provided the original author(s) and source are credited.

\section{References}

1. Ando, T., Nishio, K.: Positive selfadjoint extensions of positive symmetric operators. Tôhoku Math. J. 22, 65-75 (1970)

2. Behrndt, J., Hassi, S., de Snoo, H.S.V., Wietsma, H.L.: Monotone convergence theorems for semibounded operators and forms with applications. Proc. R. Soc. Edinb. 140, 927-951 (2010)

3. Derkach, V.A., Hassi, S., de Snoo, H.S.V.: Operator models associated with Kac subclasses of generalized Nevanlinna functions. Methods Funct. Anal. Top. 5, 65-87 (1999)

4. Derkach, V.A., Malamud, M.M.: Generalized resolvents and the boundary value problems for Hermitian operators with gaps. J. Funct. Anal. 95, 1-95 (1991)

5. Derkach, V.A., Malamud, M.M.: The extension theory of Hermitian operators and the moment problem. J. Math. Sci. 73, 141-242 (1995)

6. Donoghue, W.F.: Monotone Matrix Functions and Analytic Continuation. Springer, Berlin (1974)

7. Dym, H., McKean, H.P.: Gaussian Processes, Function Theory, and the Inverse Spectral Problem. Academic Press, New York (1976)

8. Hassi, S., Kaltenbäck, M., de Snoo, H.S.V.: Triplets of Hilbert spaces and Friedrichs extensions associated with the subclass $\mathbf{N}_{1}$ of Nevanlinna functions. J. Oper. Theory 37, 155-181 (1997)

9. Hassi, S., Kaltenbäck, M., de Snoo, H.S.V.: Generalized Kreĭn-von Neumann extensions and associated operator models. Acta Sci. Math. (Szeged) 64, 627-655 (1998)

10. Hassi, S., Langer, H., de Snoo, H.S.V.: Selfadjoint extensions for a class of symmetric operators with defect numbers (1,1). 15th OT Conference Proceedings, pp. 115-145 (1995)

11. Hassi, S., Malamud, M.M., de Snoo, H.S.V.: On Kreı̌n's extension theory of nonnegative operators. Math. Nachr. 274/275, 40-73 (2004)

12. Hassi, S., de Snoo, H.S.V.: One-dimensional graph perturbations of selfadjoint relations. Ann. Acad. Sci. Fenn. A.I. Math. 22, 123-164 (1997) 
13. Hassi, S., de Snoo, H.S.V., Szafraniec, F.H.: Componentwise and canonical decompositions of linear relations. Diss. Math. 465, 1-59 (2009)

14. Hassi, S., de Snoo, H.S.V., Winkler, H.: Boundary-value problems for two-dimensional canonical systems. Integral Equ. Oper. Theory 36(4), 445-479 (2000)

15. Hassi, S., de Snoo, H.S.V., Winkler, H.: On exceptional extensions close to the generalized Friedrichs extension of symmetric operators. Oper. Theory Adv. Appl. 175, 111-120 (2007)

16. Hassi, S., Wietsma, H.L.: Products of Nevanlinna functions with symmetric rational functions (submitted)

17. Kac, I.S.: On integral representations of analytic functions mapping the upper half-plane onto a part of itself. Uspekhi Mat. Nauk 11, 139-144 (1956)

18. Kac, I.S.: The spectral theory of a string. Ukrainian Math. J. 46 (1994), 159-182 (Russian) (English translation: Ukrainian Math. J. 46, 159-182 (1994))

19. Kac, I.S., KreĬn, M.G.: $R$-functions-analytic functions mapping the upper halfplane into itself. Supplement to the Russian edition of F.V. Atkinson, Discrete and continuous boundary problems. Mir, Moscow 1968 (Russian) (English translation: Am. Math. Soc. Transl. Ser. 2, 103, pp. 1-18 (1974))

20. Kac, I.S., Kreĭn, M.G.: On spectral functions of a string, in the Russian edition of F.V. Atkinson, Discrete and Continuous Boundary Problems. Mir, Moscow 1968 (Russian) (English translation: Am. Math. Soc. Transl. Ser. 2, 103, pp. 19-102 (1974))

21. Kaltenbäck, M., Winkler, H., Woracek, H.: Singularities of generalized strings. Oper. Theory Adv. Appl. 163, 191-248 (2005)

22. Kreı̌n, M.G.: The theory of self-adjoint extensions of semibounded Hermitian transformations and its applications I, II. Mat. Sb. 2, 431-495 (1947); 21, 365-404 (1947)

23. Langer, H., Winkler, H.: Direct and inverse spectral problems for generalized strings. Integral Equ. Oper. Theory 30, 409-431 (1998)

24. Woracek, H.: An inverse spectral theorem for Krein strings with a negative eigenvalue, Monatshefte für Mathematik, to appear 